\title{
The acute effects of aerobic exercise on sleep in patients with depression: study protocol for a randomized controlled trial
}

\author{
Gavin Brupbacher ${ }^{1,2^{*}}$ (D) Doris Straus ${ }^{2}$, Hildburg Porschke ${ }^{2}$, Thea Zander-Schellenberg ${ }^{3}$, Markus Gerber ${ }^{4}$,
} Roland von Känel ${ }^{5}$ and Arno Schmidt-Trucksäss ${ }^{1}$

\begin{abstract}
Background: Unipolar depression is one of the most important mental disorders. Insomnia is a symptom of cardinal importance in depression. It increases the risk to develop depression, negatively affects disease trajectory, is the most common symptom after remission, increases the risk of relapse, and is associated with higher suicide rates. Existing therapies for insomnia in depression have limitations. Further adjuvant therapies are therefore needed. Acute aerobic exercise has been shown to have beneficial effects on sleep in healthy individuals and patients with insomnia. We therefore hypothesize that a single session of aerobic exercise has a positive impact on sleep in patients with unipolar depression. This trial aims to investigate the effects of a single bout of aerobic exercise on the subsequent night's sleep in patients with depression.
\end{abstract}

Methods/design: This is a two-arm parallel group, randomized, outcome assessor blinded, controlled, superiority trial. Patients between 18 and 65 years of age with a primary diagnosis of unipolar depression (without a psychotic episode) are included. Exclusion criteria are regular use of hypnotic agents, opioids, and certain beta-blockers, as well as the presence of factors precluding exercise, history of epilepsy, restless legs syndrome, moderate obstructive sleep apnea, and a BMI $>40$. The intervention is a single bout of aerobic exercise, performed for $30 \mathrm{~min}$ on a bicycle ergometer at $80 \%$ individual anaerobic threshold. The control group sits and reads for $30 \mathrm{~min}$. The primary outcome is sleep efficiency measured by polysomnography. Secondary outcomes include further polysomnographic variables, subjective pre-sleep arousal, nocturnal cardiovascular autonomic modulation, subjective sleep quality, daytime sleepiness, and adverse events. According to the sample size calculation, a total of 92 patients will be randomized using minimization.

Discussion: This trial will add new information to the body of knowledge concerning the treatment of insomnia in patients with depression. Thereby, the results will inform decision makers on the utility of acute aerobic exercise.

Trial registration: Clinicaltrials.gov, NCT03673397. Protocol version 1 registered on 17 September 2018.

Keywords: Exercise, Depression, Sleep, Polysomnography, Heart rate variability, Blood pressure, Randomized controlled trial, Protocol

\footnotetext{
* Correspondence: gavin.brupbacher@unibas.ch

${ }^{1}$ Division of Sports and Exercise Medicine, Department of Sport, Exercise and

Health, University of Basel, Birsstrasse 320 B, 4052 Basel, Switzerland

${ }^{2}$ OBERWAID AG, Rorschacher Strasse 311, 9016 St. Gallen, Switzerland

Full list of author information is available at the end of the article
}

(c) The Author(s). 2019 Open Access This article is distributed under the terms of the Creative Commons Attribution 4.0 International License (http://creativecommons.org/licenses/by/4.0/), which permits unrestricted use, distribution, and

reproduction in any medium, provided you give appropriate credit to the original author(s) and the source, provide a link to the Creative Commons license, and indicate if changes were made. The Creative Commons Public Domain Dedication waiver (http://creativecommons.org/publicdomain/zero/1.0/) applies to the data made available in this article, unless otherwise stated. 


\section{Background}

Unipolar depression is a mental disorder of paramount importance. Worldwide the lifetime prevalence is estimated to be between 10 and $15 \%$ [1]. It is projected to become the leading cause of burden of disease worldwide by 2030 [2]. Core symptoms of depression are depressed mood, anhedonia, and a lack of drive. Depression is associated with an increased risk of comorbidities [3] and cardiovascular mortality [4]. It is also associated with lower cardiorespiratory fitness [5], an independent risk factor for cardiovascular mortality in healthy individuals [6].

There is a plethora of research concerning treatments for depression. Psychotherapy, pharmacotherapy or a combination of both are the primary treatments for depression according to guidelines [7, 8]. Meta-analytic data show moderate to large effect sizes for pharmacotherapy (0.35), psychotherapy (0.37), and combined therapy (0.74) when compared to placebo [9]. However, the STAR*D trial investigating four treatment steps in over 4000 patients concluded that only two-thirds of people treated for depression with pharmacotherapy, cognitive behavioral therapy, or both were in remission after treatment [10]. Moreover, the majority of patients tend to prefer non-pharmacological treatments for insomnia [11, 12] and depression [13]. Hence, there is a need for further adjuvant non-pharmacological treatment options.

Insomnia encompasses problems initiating or maintaining sleep with daytime impairments [14]. Another symptom may be non-restorative sleep. The current state of knowledge suggests that hyperarousal [15, 16], as well as sleep reactivity [17], are core etiological factors of insomnia. Hyperarousal can be cognitive (e.g., rumination, dysfunctional beliefs), emotional (emotional reactivity), cortical (beta activity in sleep EEG), or physiological (e.g., metabolic rate, heart rate variability).

There is an abundance of research related to insomnia treatments. Cognitive behavioral therapy for insomnia (CBT-I) and pharmacotherapy are currently considered first- and second-line therapies for insomnia (regardless whether comorbidities are present or not) $[17,18]$. CBT-I is very effective [19]. However, the number of trained specialists considerably limits access to this treatment. Pharmacologic agents such as benzodiazepines, nonbenzodiazepines, and sedating antidepressants are another, arguably more frequently administered therapy for insomnia. However, pharmacotherapy has multiple limitations: (1) effects have been shown to be statistically significant but of limited clinical relevance [20, 21], (2) dosages and (3) prescription duration frequently exceed recommendations of health agencies [22, 23] (especially in patients with comorbidities [24, 25]), (4) it potentially has severe adverse effects [26-28], and (5) patients often prefer non-pharmacological therapies [11, 29, 30]. Therefore, there is a need to develop further nonpharmacological treatments.

Insomnia is a highly relevant symptom of depression. Depending on the methodological approach, studies have found $25-90 \%$ prevalence rates of insomnia in people with depression [31, 32]. Longitudinal studies have repeatedly found a bidirectional link between insomnia and depression [33-36]. Insomnia is of prognostic relevance. It negatively affects the disease trajectory [37], is the most frequent residual symptom after treatment response or remission [31, 38, 39], increases the probability of relapse $[37,40,41]$, and is an independent risk factor for suicide $[42,43]$ as well as adverse somatic outcomes, particularly cardiovascular disease [44]. Insomnia has considerable economic cost. Individuals who have insomnia are at higher risk for work presenteeism $[45,46]$ and absenteeism [47] with higher direct costs per short-term absence [48]. Sleep problems are also associated with more work injuries [49]. Insomnia increases the risk of ending employment prematurely [50] and increases the risk of disability retirement due to depression [51].

Insomnia of depressed individuals has been neglected in research, despite its known relevance. Until approximately one decade ago, a central etiological distinction was made between 'organic' and 'psychogenic' or 'primary' and 'secondary' insomnia [52]. However, this distinction has been challenged because, among other reasons, there is often a lack of evidence for a mechanistic distinction between primary and secondary insomnia [52]. Therefore, a paradigm shift has become apparent, recommending specific treatments for comorbid insomnia. This paradigm shift might explain why, until recently, most trials have focused on patients with insomnia without any comorbidities.

Aerobic exercise is a viable candidate for the treatment of insomnia in patients for depression. Meta-analyses have shown a positive impact of acute and chronic exercise in healthy individuals with small to moderate effect sizes [53, 54]. Meta-analyses focusing on individuals with at least mild insomnia but no comorbidities have found a moderate effect of chronic aerobic exercise on sleep quality [54-56]. Aerobic exercise has further positive effects such as improving depressive symptoms [57] and cardiorespiratory fitness [58]. The latter is especially relevant for the reduction of cardiovascular risk [6].

Current sleep hygiene recommendations, which are also relevant for depression, lack feasibility. In particular, they state that exercise should not be performed after $2 \mathrm{pm}$ [59]. This time constraint presents a considerable limitation since many people can only accommodate aerobic exercise in the late afternoon or evening. Even more so this may be limiting for patients with morning depression 
who may feel more energetic to exercise in the afternoon [60]. However, a recent meta-analysis has found equivocal effects of exercise performed in the afternoon or evening in healthy individuals [54]. In healthy individuals, effects of a single evening bout of aerobic exercise on nocturnal heart rate variability seem to depend on intensity, duration, and timing relative to sleep but do not seem to alter subjective sleep quality $[61,62]$. One trial has investigated the effects of acute aerobic exercise in chronic primary insomniacs, showing moderate to large effect sizes for shortened sleep onset latency, improved sleep efficiency, and longer total sleep time [63].

\section{Rationale and hypotheses}

An extensive literature search yielded no randomized controlled trials investigating the acute effects of exercise on sleep in patients with depression. Several studies concerned with chronic effects are available, and we will summarize these in our upcoming systematic review and network meta-analysis (PROSPERO ID 115705, registration not published yet). Considering this gap in the literature and the uncertainty concerning the effects of exercise performed in the afternoon on sleep in patients with depression, a trial on this topic is of high clinical importance.

We hypothesize that an acute bout of aerobic exercise improves 1) sleep efficiency [54,63], 2) sleep continuity [63], 3) sleep architecture [54], 4) subjective sleep quality $[64], 5)$ daytime sleepiness [65], 6) nocturnal blood pressure [66], 7) pre-sleep arousal, and 8) pre- and postsleep heart-rate variability. We expect no effect on 9) nocturnal heart rate variability [62] and 10) the frequency and severity of adverse events [64].

This paper presents the design and protocol for the trial according to the Standard Protocol Items: Recommendations for Interventional Trials (SPIRIT) statement [67] (Additional file 1).

\section{Methods/design}

\section{Study design and setting}

This study is designed as a two-arm parallel group, randomized, outcome assessor blinded, controlled trial, to assess the superiority of (i) an acute bout of aerobic exercise compared to (ii) control in ameliorating sleep efficiency in patients with depression (Fig. 1).

The trial is conducted in the psychosomatic inpatient rehabilitation unit of the OBERWAID AG, a rehabilitation clinic in St. Gallen, Switzerland. Patients are referred to the clinic by their general practitioner or a psychiatrist. On average, approximately 250 patients with a primary diagnosis of an ICD-10 depressive episode without psychotic features are referred to the clinic annually. The OBERWAID AG also offers outpatient psychosomatic care, cardiovascular inpatient rehabilitation, and orthopedic aftercare.

\section{Participants}

\section{Eligibility criteria}

Patients aged 18-65 years with a diagnosis of depression (confirmed by experienced psychiatrists according to ICD-10) undergoing inpatient psychosomatic rehabilitation in the OBERWAID clinic are eligible for inclusion.

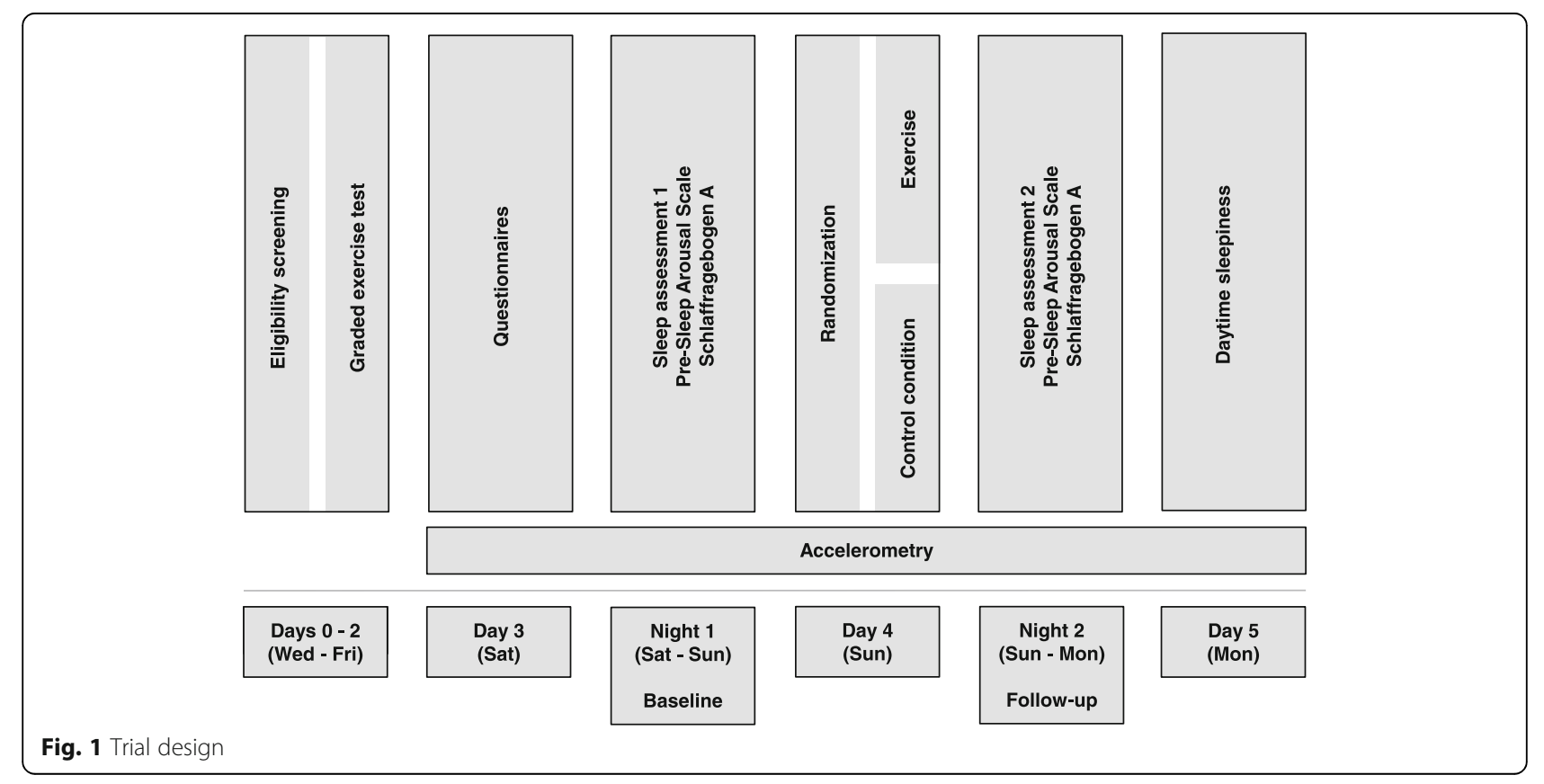


Further inclusion and exclusion criteria with corresponding rationales are listed in Table 1.

\section{Recruitment}

Patients are given the study information at the preliminary medical consultation, which takes place approximately 3 weeks (but no later than 2 days) before admission. On the

Table 1 Inclusion and exclusion criteria

\begin{tabular}{ll}
\hline Criterion & Rationale \\
\hline Inclusion criteria & \\
$\geq 18$ and $\leq 65$ years old & $\begin{array}{l}\text { Results of the trial should be } \\
\text { generalizable to the working age } \\
\text { population. Furthermore, there can } \\
\text { be numerous reasons for sleep } \\
\text { disorders in older patients [68] }\end{array}$ \\
& $\begin{array}{l}\text { Mental disorder used to define the } \\
\text { (F32, F33) without psychotic } \\
\text { episode according to ICD-10 }\end{array}$
\end{tabular}

\section{Exclusion criteria}

Regular use of hypnotic agents* (patients are included if no hypnotic agents were taken two weeks before study

participation)

Factors precluding exercise testing or training

Use of beta-blockers (except carvedilol and nebivolol)

Use of opioids

History of epilepsy

Restless legs syndrome defined by $\geq 7$ points on the restless legs screening questionnaire [76]

Moderate or severe sleep apnea defined by an oxygen desaturation index $(\mathrm{ODI}) \geq 15$ in the first polysomnography

Morbid adiposity with BMI $>40$

Use of hypnotic agents might mask the effect of aerobic exercise on sleep

For safety reasons, patients who have any condition which precludes exercise testing or training are excluded. Absolute and relative contraindications are based on ACSM's Guidelines for Exercise Testing and Prescription [69]

Except for carvedilol and nebivolol $[70,71]$, beta-blockers have been shown to reduce nocturnal melatonin levels $[70,72,73]$

Opioids affect sleep architecture [74]

Epilepsy is associated with quantitative and qualitative alterations of sleep [75] and might, therefore, mask the intervention effects

Can also cause sleep disturbance, but the etiology is distinct from depression [77]

Sleep apnea is a distinct sleep disorder with clearly delineated etiology. ODI has been shown to be highly correlated with the apnea-hypopnea index and to detect sleep apnea with high sensitivity and specificity [78-80]

These patients might suffer from hypoventilation syndrome which affects sleep

ICD-10 International Classification of Diseases, version 10, ODI oxygen desaturation index, $B M I$ body mass index

*Hypnotic agents are defined as follows: orexin receptor agonists, benzodiazepine receptor agonists, sedating antidepressants, neuroleptics, benzodiazepines, melatonin agonists, heterocyclics, anticonvulsants, over the counter sleep aids (sedating antihistamines, melatonin L-tryptophan, valerian), and cannabinoids day of admission, the clinical trial is presented to the patients by the study coordinator. Patients who are interested in participating in the study can ask the study coordinator questions directly. In order to ensure adequate time to consider their participation, interested and potentially eligible patients meet the study coordinator for an informed discussion again on the following day (i.e., the first day after admission). The study coordinator obtains the written informed consent from patients willing to participate. Should the study coordinator be absent, his designated replacement obtains the informed consent. The information sheet and consent form are in German (Additional file 2). It is clinical routine for the leading physicians to give all patients a general consent form.

\section{Retention}

Appointments for data collection are included in the therapy schedule during the first 6 days of rehabilitation, which should reduce the burden on patients and ensure protocol fidelity. We give patients feedback on their measurements. We will send a digital copy of publications based on this trial to participants if they have chosen to receive one. No monetary compensation is offered for participation.

\section{Withdrawal, discontinuation, and stopping rules}

Participants can withdraw from the trial at any time without having to provide reasons. If possible, we collect follow-up data and reasons for withdrawal. Patients can be withdrawn from the trial by the principal investigator for medical reasons (e.g., transfer to another hospital). Exercise testing and training is immediately stopped, should any indications for exercise test termination, as defined by the American College of Sports Medicine, be met [69]. In this case, we collect follow-up data if there are no contraindications.

\section{Measurements and procedure Rationale for measurements}

This section offers a rationale for the selected variables and performed measurements. Details of each assessment (measurement variable, reliability, validity, analysis metric, method of aggregation, time point, and clinical relevance) are provided below.

Baseline This trial does not exclude patients with psychiatric comorbidities in order to preserve external validity. Therefore, we perform an extensive baseline characterization of the sample (Fig. 2). The characterization consists of questionnaires on psychiatric, somatic, and insomnia-specific symptom severity as well as sleep-related variables such as chronotype and sleep-related cognitions. Furthermore, baseline 


\begin{tabular}{|c|c|c|c|c|c|c|}
\hline & \multicolumn{6}{|c|}{ STUDY PERIOD } \\
\hline & Screening & Background & Baseline & Allocation & Intervention & Follow-up \\
\hline TIMEPOINT $^{\star *}$ & $-t_{3}$ & $-t_{2}$ & $-t_{1}$ & $t_{0}$ & $t_{1}$ & $t_{2}$ \\
\hline Days after admission & 0.2 & 3 & 3.4 & 4 & 4 & $4 \cdot 5$ \\
\hline \multicolumn{7}{|l|}{ ENROLMENT: } \\
\hline Information & $\mathrm{x}$ & & & & & \\
\hline Informed consent & $x$ & & & & & \\
\hline \multirow[t]{2}{*}{ Eligibility screening } & $x$ & & & & & \\
\hline & & & & $x$ & & \\
\hline \multicolumn{7}{|l|}{ INTERVENTIONS: } \\
\hline \multirow[t]{2}{*}{ Aerobic exercise } & & & & & $x$ & \\
\hline & & & & & $x$ & \\
\hline \multicolumn{7}{|l|}{ ASSESSMENTS: } \\
\hline \multirow{3}{*}{$\begin{array}{l}\text { Graded exercise test * } \\
\text { Patient Health Questionnaire } \\
\text { (PHQ-9) } \\
\text { Hospital Anxiety \& } \\
\text { Depression Questionnaire* }\end{array}$} & $x$ & & & & & \\
\hline & & $\mathrm{x}$ & & & & \\
\hline & & $x$ & & & & \\
\hline \multirow{3}{*}{$\begin{array}{l}\text { Perceived Stress Scale } \\
\text { Patient Health Questionnaire } \\
\text { (PHQ-15)* } \\
\text { Cumulative Illness Rating } \\
\text { Scale* }\end{array}$} & & $\mathrm{x}$ & & & & \\
\hline & & $x$ & & & & \\
\hline & & $x$ & & & & \\
\hline \multirow{2}{*}{$\begin{array}{l}\text { Epworth Sleepiness Scale } \\
\text { Pittsburgh Sleep Quality }\end{array}$} & & $x$ & & & & \\
\hline & & $\mathrm{x}$ & & & & \\
\hline \multirow{4}{*}{$\begin{array}{l}\text { Dysfunctional beliefs \& } \\
\text { attitudes about sleep scale } \\
\text { Ford Insomnia Response to } \\
\text { Stress Test } \\
\text { Morningness-Eveningness } \\
\text { Questionnaire } \\
\text { Expectancy and Credibility } \\
\text { (single items) }\end{array}$} & & $x$ & & & & \\
\hline & & $x$ & & & & \\
\hline & & $x$ & & & & \\
\hline & & $\mathrm{x}$ & & & & \\
\hline Polysomnography* & & & $X^{*}$ & & & $x$ \\
\hline \multirow{3}{*}{$\begin{array}{l}\text { Pre-Sleep Arousal Scale } \\
\text { Schlaffragebogen A, } \\
\text { 'subjective sleep scale' } \\
\text { Stanford Sleepiness Scale }\end{array}$} & & & $x$ & & & $x$ \\
\hline & & & $x$ & & & $x$ \\
\hline & & & & & & $x$ \\
\hline \multirow{4}{*}{$\begin{array}{l}\text { Rate of perceived exertion } \\
\text { Befindlichkeitsskala, } \\
\text { 'mood scale' } \\
\text { Actigraphy }\end{array}$} & & & & & $x$ & \\
\hline & & & & & $x$ & \\
\hline & & & & & $x$ & \\
\hline & & 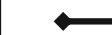 & & & & \\
\hline
\end{tabular}

Fig. 2 Participant timeline - Spirit figure

polysomnography will provide an extensive characterization of sleep.

Primary outcome Sleep disorders are of complex etiology and have various psycho-physiological consequences. Therefore, it is recommended to measure the effects of interventions on insomnia symptoms as well as other factors such as daytime functioning and mood [81]. Insomnia can be classified into sleep-onset, sleep-maintenance, and sleep-offset 
subtypes. Meta-analysis of polysomnographic data has shown that patients with depression and other mental health disorders have mixed (i.e., sleep-onset and sleepmaintenance) insomnia [82]. Sleep efficiency best captures both of these aspects and is therefore chosen as the primary outcome. Objective quantification of the primary outcome is vital in this study since the blinding of participants is not possible, thereby potentially affecting subjective measurements.

Secondary outcomes Secondary outcomes which inform clinical decision making include further polysomnographic variables, subjective pre-sleep arousal, cardiovascular autonomic modulation, subjective sleep quality, daytime sleepiness, and adverse events. Wake after sleep onset and number of awakenings identify sleep-maintenance insomnia, while sleep-onset latency characterizes sleep-onset insomnia.

Patients with depression show characteristic changes in rapid eye movement (REM) latency (time between sleep onset and first rapid eye movement sleep episode), REM-density (frequency of rapid eye movements/REM episode), and duration of REM sleep (collectively known as REM pressure) [82]. More importantly, these alterations have a negative impact on treatment and increase the risk of relapse [83]. Exercise has been shown to reduce REM sleep and increase slow wave sleep [54]. The discrepancy between objective and subjective sleep measurements is well documented [81, 84, 85]. Therefore, we measure the subjective sleep quality of the previous night in addition to the objective sleep assessment. Patient views influence the treatment of sleep disorders $[86,87]$. Hence, it is crucial to ascertain the credibility of the intervention and the expectancy of participants. Adverse events are underreported in sleep trials but highly relevant to clinical decision making [88]. The case report form specifically captures adverse events (see below) to gauge the benefit to harm ratio.

\section{Screening}

After providing informed consent, we formally screen patients for inclusion and exclusion criteria of this study. They are consulted by an experienced psychiatrist and undergo a full history and medical examination by an experienced internist, including vital parameters. (Resting electrocardiogram may be forgone if the general practitioner of the patient provides one no older than 2 months.) Patients with undiagnosed sleep apnea are excluded according to the baseline polysomnography (see below).

\section{Graded exercise testing}

Patients fulfilling all eligibility criteria (except the sleep apnea criterion, which is determined later by polysomnography) undergo sub-maximal graded exercise testing on a bicycle ergometer (ergoselect 200, Ergoline, Bitz, Germany). The goal is to determine the individual anaerobic threshold. The anaerobic threshold is used to standardize the exercise intensity of the intervention across patients. Since subjects vary in their endurance capacity and weight, we adjust initial and subsequent work rates (Watts) between subjects before the test. Stage duration is always 3 minutes, as this has been found to yield the most reliable and valid results [89]. We measure blood lactate at the end of each stage using capillary blood from the earlobe. We assess blood lactate level with Lactate Scout+ (SensLab GmbH, Leipzig, Germany), a validated hand-held analyzer [90]. Heart rate is measured using the validated Polar ${ }^{\circ}$ H7 chest strap (Polar OY, Finland) [91]. Parasympathetic drive is still present at lower exercise intensities and thus increases heart rate variability [92]. The average heart rate during the last $30 \mathrm{~s}$ of each stage is extracted to improve precision and used for further analyses. Ratings of perceived exertion are recorded at the end of each stage according to Borg's 6-20 scale [93]. The graded exercise test data are analyzed using a specialized software program (Ergonizer, Freiburg, Germany). The individual anaerobic threshold is determined according to the method of Dickhuth et al. [94].

\section{Baseline characterization of symptom severity}

To characterize the study population in detail, we will report multiple continuous measures at baseline as median with corresponding interquartile range or frequencies.

Somatic and psychological symptom severity In accordance with the International Consortium for Health Outcomes Measurement (ICHOM) Depression and Anxiety working group, depression symptoms are assessed with the German version of the Patient Health Questionnaire-9 (PHQ-9) including the additional question on functioning [95]. The nine symptom items are scored on a four-point Likert scale (not at all to nearly every day; Cronbach's $\alpha=0.89$ ). The cut-offs of the aggregated sum score have been validated and allow for classification of mild to severe depression [96]. Anxiety is assessed using the Hospital Anxiety and Depression Scale. This questionnaire measures depression and anxiety with seven items each on a four-point Likert scale [97]. A meta-analysis has found Cronbach's $\alpha$ to be 0.83 and 0.82 for the anxiety and depression subscales, respectively [98]. Diagnostic test accuracy of the depression and anxiety subscale is high [99]. More recently, a meta confirmatory factor analysis has suggested the presence of a general distress factor explaining most of the covariance between items [100]. The German version has been shown to have adequate psychometric properties [101]. We measure stress with the German version of the ten-item Perceived 
Stress Scale, which has demonstrated good reliability (Cronbach's $\alpha=0.84$ ) and validity [102]. Therein, stress is operationalized as the degree to which life is experienced as unpredictable, uncontrollable, and overloaded in the past months on a five-point Likert scale (never to very often). Somatic multimorbidity is measured using the self-administered Patient Health Questionnaire Somatic Symptom Scale (PHQ-15), which has good reliability (Cronbach's $\alpha=0.8$ ) and validity [103]. This questionnaire measures the presence and severity of somatic symptoms during the past 4 weeks on a three-point Likert scale (not bothered at all to bothered a lot). The items assess symptoms clusters which account for more than $90 \%$ of physical complaints reported in out-patient settings [103]. Lastly, the German version of the Modified Cumulative Illness Rating Scale provides physician-rated scores of multimorbidity. This scale has a good inter-rater agreement and concurrent and predictive validity. It measures the presence and severity multimorbidity over 14 organ systems on a five-point scale (no problem to extremely severe problem) [104].

Subjective measurements related to sleep Multiple sleep-related variables are measured to characterize the impact of sleep disorders in the population. The German version of the Pittsburgh Sleep Quality Index [105] is used to quantify subjective sleep disturbance. This 18 item scale assesses subjective sleep quality, sleep latency, sleep duration, habitual sleep efficiency, sleep disturbances, use of sleeping medication, and daytime dysfunction. It has adequate psychometric properties (Cronbach's $\alpha=0.83$ ) [106]. The corresponding global score with a cut-off value of $\geq 5$ has been shown to distinguish good and poor sleepers [107]. We measure sleep reactivity, i.e., the degree to which an individual experiences disrupted sleep due to stress, with the Ford Insomnia Response to Stress Test [108, 109]. The nine-item self-report questionnaire is answered on a four-point Likert scale (not very likely to very likely) with higher total scores indicating a higher likelihood of stress-induced insomnia. The reliability (Cronbach's $\alpha=0.80$ ) and validity of the German version have been demonstrated [110]. Dysfunctional sleep-related thoughts and attitudes are related to the development and trajectory of sleep disorders [111]. The reliability and validity of the German short form of the dysfunctional beliefs and attitudes about sleep scale have recently been demonstrated (average Cronbach's $\alpha=0.71$ ) [112]. Sixteen items measure four domains (consequences, worry/helplessness, expectations, medication) on a ten-point Likert scale (strongly disagree to strongly agree). Excessive daytime sleepiness is associated with insomnia and depression $[113,114]$ as well as reduced quality of life and work performance [115]. We assess chronic daytime sleepiness using the German version of the Epworth Sleepiness Scale. This questionnaire has good reliability (Cronbach's $\alpha 0.83$ in patients) and validity [116]. The likelihood of dozing off in eight daily situations (e.g., sitting and reading) is assessed on a four-point Likert scale (would never doze to high chance of dozing). Chronotype, i.e., peak alertness throughout the day, is associated with more severe depression, insomnia, and suicidality in patients with major depression. Furthermore, eveningness substantially increased the risk of non-remission independently of insomnia severity [117]. The MorningnessEveningness Questionnaire assesses chronotype with 19 multiple-choice questions (four- or five-point scale) on sleep habits and propensity for performance throughout the day. The sum ranges from 16 to 86 and can be translated into chronotypes ( $<42$, evening type; $42-58$, neither; $>58$, morning type) [118]. Criterion validity has been established [119]. Validity and reliability (test-retest reliability $>0.96$ ) have been confirmed for the German version [120].

\section{Polysomnography (baseline and follow-up)}

Polysomnography is considered the gold standard of sleep assessment [121]. However, one significant drawback of this method is the so-called first-night effect. This effect describes altered sleep patterns due to novel environments, disturbances by measurement equipment, a potential Hawthorne effect, or a combination thereof [122]. Such alterations typically represent worse sleep quality, e.g., reduced sleep efficiency, in the first compared to the second night [122]. Although this effect has been found in different patient groups, it is clearly attenuated in individuals with depression [123-125]. Moreover, polysomnographic measurements are expensive and performing multiple measurements is not always feasible. Several authors argue that data from the first night (i.e., baseline) should, therefore, be used in the analyses $[123,125]$. In designs where baseline and followup data are collected, analysis of covariance (ANCOVA) has multiple advantages. Firstly, there is less potential for bias compared to the analysis of change (i.e., pre minus post) scores or follow-up data only. Secondly, ANCOVA has higher statistical power $[126,127]$.

Montage Polysomnography is performed with the SOMNOscreen $^{\text {tox }}$ plus RC (Somnomedics, Randersacker, Germany) using the following montage: one EEG channel (Fp2-A1, $512 \mathrm{~Hz})$, two EOG channels $(1 \mathrm{~cm}$ below and 1 $\mathrm{cm}$ lateral of the outer right canthus as well as $1 \mathrm{~cm}$ above and $1 \mathrm{~cm}$ lateral of the outer left outer canthus, $512 \mathrm{~Hz}$ ), one EMG channel (Chin1-Chin2, $512 \mathrm{~Hz}$ ), one ECG channel (modified lead II, $512 \mathrm{~Hz}$ ), thoracic respiratory effort channel (inductance plethysmography belt, $32 \mathrm{~Hz}$ ), finger photoplethysmography (non-dominant arm, $128 \mathrm{~Hz}$ ), 
body position (stored every $30 \mathrm{~s}$ ), movement $(32 \mathrm{~Hz})$, and ambient light (stored every $30 \mathrm{~s}$ ). Relevant skin areas are prepared with Nuprep (Weaver \& Co., Aurora, CO, USA) before electrode placement to reduce skin impedance. Although this montage does not comply with AASM standards [128], the use of a single channel EEG, two EOG channels, and one EMG channel has been shown to have good validity when assessing sleep stages [129].

Analysis of polysomnography We apply low- and highfrequency filters according to AASM guidelines [128]. Oxygen desaturation index (ODI) is defined as the number of oxygen desaturations $(\geq 4 \%)$ measured by photoplethysmography per hour of total sleep time [128]. We analyze polysomnography data visually in epochs of $30 \mathrm{~s}$ by two trained, independent, and blinded raters according to AASM guidelines [128]. If sleep efficiency differs by $\leq 5 \%$ between raters, we use the mean of each quantitative sleep parameter of both raters for analysis. If sleep efficiency differs by $>5 \%$ between raters, a consensus scoring is done by a third rater who is blinded against allocation and previous ratings. Inter-rater reliability (intraclass correlation) based on the scoring of the first two raters will be provided for the primary outcome. Sleep parameters are calculated with the proprietary DOMINO software (Somnomedics, Randersacker, Germany) (Table 2).

Sleep parameters can be grouped into three domains:

1. Sleep continuity: high sleep efficiency, low sleep onset latency, low wake after onset, low number of awakenings

2. Sleep depth: less stage 1 and 2 sleep, more stage 3 (i.e., slow wave) sleep

3. REM pressure: low REM latency, more REM sleep, (high REM-density)

Nocturnal autonomic cardiovascular modulation Sleep hygiene recommendations include daily exercise. Two caveats of this recommendation are that exercise should be performed before $2 \mathrm{pm}$ and that no strenuous exercise ought to be done close to bedtime. One reason for this recommendation is that exercise in the afternoon or evening might increase arousal and thereby prevent sleep [59]. However, evidence from numerous epidemiological, observational, and experimental studies have repeatedly failed to show such an adverse effect or have found that the opposite is true $[54,130]$. The issue of nocturnal autonomic modulation following exercise is of central importance for three reasons. Firstly, arousal is integral to one of the most widely accepted etiological theories of insomnia $[15,16]$. Secondly, heart rate variability (HRV), as a marker of autonomic arousal, has been proposed as a
Table 2 Sleep parameters

\begin{tabular}{ll}
\hline Sleep parameter & Definition \\
\hline $\begin{array}{l}\text { Total recording } \\
\text { time (TRT) }\end{array}$ & $\begin{array}{l}\text { Time between the lights-off and } \\
\text { lights-on markers (min) }\end{array}$ \\
Total sleep time (TST) & $\begin{array}{l}\text { Time asleep (in any sleep stage) } \\
\text { within TRT (min) } \\
\text { TST = N1 + N2 + N3 + REM }\end{array}$ \\
Sleep onset & Time between lights-off marker \\
latency (SOL) & and first epoch of any sleep stage \\
Wake after sleep & Time awake after first sleep episode (min) \\
onset (WASO) & WASO = TRT - SL - TST \\
Number of & Number of wake periods of at least \\
awakenings (NA) & two epochs after sleep onset \\
Sleep efficiency (SE) & $\begin{array}{l}\text { Percentage of sleep while in bed (\%) } \\
\text { SE = (TST / TRT) } \times \text { 100 }\end{array}$ \\
N1 & Stage 1 (in minutes and \% TST) \\
N2 & Stage 2 (in minutes and \% TST) \\
N3 & Stage 3 (in minutes and \% TST) \\
Light sleep & Stage 1 and 2 (in minutes and \% TST) \\
NREM (non-REM) sleep & Stage 1-3 (in minutes and \% TST) \\
REM & Rapid eye movement (in minutes and \% TST) \\
REMLAT & Time between sleep onset and the \\
occurrence of the first REM sleep epoch (min) & Number of transitions between any wake \\
Stage shift index & or sleep stage/hours of sleep \\
\hline
\end{tabular}

potential pathophysiological mechanism linking depressive disorders [131, 132] and insomnia [133] with cardiovascular disease. Thirdly, nocturnal blood pressure and nocturnal blood pressure dipping are of prognostic relevance for cardiovascular disease [134]. Therefore, sleep quality, as well as nocturnal autonomic activity, should be assessed when evaluating interventions in sleep research. Measurements of heart rate, HRV, and pulse transit time (and thereby calculated blood pressure) are collected to quantify the effect of exercise on parameters of autonomic cardiovascular arousal.

We record the ECG while subjects are lying in bed. Patients were instructed to lie in the supine position and refrain from speaking as well as moving during the two short-term (i.e., 5-minute) recordings. No instructions concerning breathing were given. All ECG measurements were sampled at $512 \mathrm{~Hz}$ using modified lead II.

Three sections of HRV data are analyzed:

1. A short-term pre-sleep measurement with a duration of $5 \mathrm{~min}$, beginning after lights off, is taken to quantify pre-sleep autonomic modulation. Measurements in which patients have fallen asleep are excluded from the analysis.

2. A nocturnal 6-h segment beginning after sleep onset. We choose this definition of the nocturnal HRV assessment for multiple reasons. According 
to guidelines, durations of recordings have to be of equal length [135]. Nocturnal measurement segments of 4 or $6 \mathrm{~h}$ are frequently used in studies $[136,137]$. In such trials, the starting point of the measurement period is usually defined by either sleep onset (identified by EEG or sleep diary) or a fixed time (e.g., 00:00). Since sleep onset is associated with increased parasympathetic modulation [138], we use sleep onset defined by polysomnography to mark the starting point of the segment to be analyzed. Using this starting point avoids potential bias which might be introduced by inter-individual differences in sleep onset latency and chronotype when using a fixed time. We chose a 6-h segment as it offers a more extended measurement period than $4 \mathrm{~h}$. Moreover, $6 \mathrm{~h}$ is commonly considered the cut-off for objective short sleep duration $[139,140]$ and thus can be understood as a minimum sleep requirement for most individuals. Nocturnal HRV is dynamic, as non-REM sleep (primarily during the first half of the night) and REM sleep (primarily during the second half of the night) are characterized by parasympathetic and sympathetic predominance, respectively $[138,141]$. We split the 6 -h period into hourly segments, which allows the dynamic mentioned above to be partially captured.

3. A short-term post-sleep segment of 5 min beginning after the last awakening and before standing up to quantify post-sleep autonomic modulation. Patients are instructed to lie supine in bed after awakening for 5 min (using a timer). The post-sleep measurement has the advantage of limiting external factors which influence HRV.

The time- and frequency-domain measures which we calculate are described in Table 3. Low (LF) and high frequency (HF) power in normalized units (LFnu and HFnu) will not be reported. The rationale for this deviation from guidelines is that LFnu, HFnu, and LF/HF ratio have been shown to carry algebraically and therefore physiologically redundant information [142, 144]. Reporting of data collection, analysis, cleaning, and calculation will follow the GRAPH guidelines [145]. The percentage of beats identified as artifacts will be reported for both groups for each segment (median and inter-quartile range). Measurements with $>5 \%$ artifacts will be excluded from analysis.

An ongoing debate in the literature concerns the choice of method for spectral analysis. Fast Fourier transform (FFT) using Welch's periodogram and autoregressive modeling (AR) seem to be the most frequently used methods for power spectral analysis [135, 143]. Another method to estimate power spectral density is the Lomb-Scargle periodogram (LSP) [146, 147]. LSP has numerous advantages when compared to FFT and AR. LSP is designed to estimate the power density spectrum directly from the unevenly sampled tachogram. AR and FFT, on the other hand, need to be interpolated and resampled to fulfill the prerequisite of evenly sampled data. Resampling leads to over- and under-estimation of LF and HF, respectively $[148,149]$. FFT and AR require a

Table 3 HRV parameters

\begin{tabular}{|c|c|c|c|}
\hline Method & Measure of variability & Calculation of variable & Physiological mechanism \\
\hline \multicolumn{4}{|l|}{ Time-domain methods } \\
\hline \multirow[t]{3}{*}{ Statistical } & RMSSD & $\begin{array}{l}\text { Root mean square of successive } \\
\text { differences of NN intervals }\end{array}$ & $\begin{array}{l}\text { Short-term components of HRV, } \\
\text { vagal modulation }\end{array}$ \\
\hline & SDNN & $\begin{array}{l}\text { Standard deviation of the of all } \\
\text { NN intervals }\end{array}$ & $\begin{array}{l}\text { Overall HRV, cyclic components } \\
\text { responsible for HRV }\end{array}$ \\
\hline & SDANN & $\begin{array}{l}\text { Standard deviation of the averages } \\
\text { of NN intervals in all 5-min segments } \\
\text { of the entire recording (only for } \\
\text { nocturnal HRV) }\end{array}$ & Long-term components of HRV \\
\hline \multicolumn{4}{|l|}{ Frequency domain methods } \\
\hline \multirow[t]{4}{*}{$\begin{array}{l}\text { Lomb-Scargle Periodogram } \\
\text { and } \\
\text { Fast Fourier transformation }\end{array}$} & $\mathrm{TP}$ & $\begin{array}{l}\text { Total power: power density spectrum } \\
\text { in the frequency range of } 0.00001 \\
\text { to } 0.4 \mathrm{~Hz}\left[\mathrm{~ms}^{2}\right]\end{array}$ & Overall HRV \\
\hline & LF & $\begin{array}{l}\text { Low-frequency power: power density } \\
\text { spectrum in the frequency range } \\
\text { of } 0.04 \text { to } 0.15 \mathrm{~Hz}\left[\mathrm{~ms}^{2}\right]\end{array}$ & $\begin{array}{l}\text { Sympathetic and vagal activity, } \\
\text { baroreflex activity (vasomotor tone) }\end{array}$ \\
\hline & $\mathrm{HF}$ & $\begin{array}{l}\text { High-frequency power: power density } \\
\text { spectrum in the frequency range } \\
\text { of } 0.15 \text { to } 0.40 \mathrm{~Hz}\left[\mathrm{~ms}^{2}\right]\end{array}$ & Vagal modulation \\
\hline & LF/HF & Ratio LF $\left[\mathrm{ms}^{2}\right] / \mathrm{HF}\left[\mathrm{ms}^{2}\right]$ & Sympathetic and vagal modulation \\
\hline
\end{tabular}


trade-off between frequency resolution and time resolution (statistical stability) when choosing window length [150] and model order [151], respectively. In contrast, LSP makes no assumptions of models. Investigations have shown that LSP is more accurate [152-154], is less noisy [154], has higher reliability [155], and is more sensitive to physiological changes [155-157] when compared to FFT. Consequently, multiple authors have suggested LSP as the method of choice for spectrum analysis of HRV [152, 158]. For these reasons, we estimate power spectral density using LSP smoothed with a moving average filter (width $0.02 \mathrm{~Hz}$ ) in this trial. To enable comparison with other studies, we also calculate Welch's FFT and will report it as a sensitivity analysis.

We perform ECG pre-processing and HRV analysis using Kubios HRV (University of Eastern Finland, Kuopio, Finland) [159]. This software has been shown to have perfect intra-class correlation coefficients $(\mathrm{ICC}=1.000)$ across HRV variables measured during different postures when compared to two other software [120]. QRS detection is based on the Pan-Tompkins algorithm [160], including bandpass filtering. Beat detection is visually inspected. Erroneously detected RR fiducial points are corrected by manual editing of R-wave. Artifacts are identified using the automatic artifact correction algorithm [161]. This algorithm has been shown to detect ectopic beats with an accuracy of $97 \%$. Ectopic beats are replaced by phantom beats using cubic spline interpolated RR values. Correction of aberrant RR intervals using cubic spline interpolation has been shown to perform as well as other correction methods for frequency analysis [162]. Detrending, i.e., removal of slow-trend and non-linear trend components is performed using the smoothness priors approach [163] with $\lambda=500$ and $\mathrm{f}_{\mathrm{c}}=0.035 \mathrm{~Hz}$ (thus not affecting the lower band of LF). Frequency-domain variables estimated by LSP are based on de-trended RR series. Frequency-domain variables estimated by FFT with Welch's periodogram are based on de-trended as well as interpolated (i.e., resampled) RR series. We employ the following parameters for power spectral density estimation using FFT. RR series are resampled to obtain an evenly sampled time series using a cubic spline interpolation with a rate of $4 \mathrm{~Hz}$. A Hann window with a width of $60 \mathrm{~s}$ and $50 \%$ overlap is used (corresponding to 240 samples). These window parameters are chosen to balance the requirement of stationarity and frequency resolution resulting in an average of 9 and 119 FFT spectra for $5 \mathrm{~min}$ and hourly segments, respectively, with a frequency resolution of $0.025 \mathrm{~Hz}$. Singh et al. [150] have shown that these approximate parameters produce a good spectral estimate (smooth with clear peaks).

Pulse transit time is calculated using ECG and pulse waveform from photoplethysmography. Blood pressure (BP) is calculated using the pulse transit time. This method has been validated [164] according to the European Society of Hypertension International Protocol (ESH-IP) revision 2010 criteria [165]. Accordingly, we perform a single initial calibration measurement (manual cuff-based method, contralateral arm of photoplethysmography, sitting position). Two significant advantages of this method are the continuous measurement of BP and elimination of cuff inflations. The latter is poorly tolerated by patients, causes awakenings, and may affect the validity of BP measurements [166]. BP levels differ between nonREM and REM sleep [167]. Therefore, mean systolic, mean diastolic, and mean arterial pressures will be reported separately for total sleep time, non-REM sleep, and REM sleep.

\section{Subjective sleep-related measurements}

Subjective pre-sleep arousal has been shown to be increased in primary insomnia [168] and seems to partially mediate the relationship between depressive symptoms and daytime fatigue [169]. We use the German version of the Pre-Sleep Arousal Scale to assess cognitive and somatic pre-sleep arousal. Eight and seven items load onto the factors somatic (Cronbach's $\alpha=0.80$ ) and cognitive (Cronbach's $\alpha=0.94$ ) arousal, respectively [170]. Items are scored on a five-point Likert scale (not at all to extremely) and summed up for each factor separately.

We measure subjective sleep quality of the baseline and post-intervention night using the revised Schlaffragebogen $A$, as recommended by guidelines [171]. Twenty-five items load onto five factors: sleep quality, recuperation after sleep, calmness before sleep, exhaustion before sleep, and psychosomatic symptoms during sleep. Internal consistency, factor structure, and validity have been demonstrated in numerous populations [172].

The clinical relevance of excessive daytime sleepiness is highlighted above (cf. rationale for ESS questionnaire). State sleepiness is recorded four times $(0800,1200,1600$, $2000 \mathrm{~h}$ ) on the day after the experimental condition to gauge the effects on daytime somnolence. The Stanford Sleepiness Scale is a single item questionnaire assessing the degree of momentary sleepiness on a seven-point scale [173]. Adequate psychometric properties have been demonstrated [174].

\section{Expectancy and credibility}

Insomnia treatment guidelines stress the importance of contextual factors such as patient preference and satisfaction when choosing the most suitable therapy $[88,175]$. Credibility can increase expectancy [176]. The latter has been shown to influence outcomes in depression and other disorders [177]. Since patients cannot be blinded in exercise studies, it is especially important to consider expectancy. We assess credibility and expectancy on day three (i.e., before randomization) using two items (adapted 
from [178, 179]): "At this point, how logical does the therapy offered to you seem?", "At this point, how successfully do you think this treatment will be in reducing your insomnia symptoms?". Patients rate these items on a four-point Likert scale (not at all to very).

\section{Randomization and blinding}

A non-deterministic minimization algorithm is used to assign interventions. Allocation to intervention or control group is done using the open source software for online minimization (Oxford Minimization and Randomization, OxMaR) [180]. All necessary data for minimization is collected using surveys integrated into the eCRF. The required data are entered in the web-based randomization software by the study nurses. Upon confirmation that the data are correct, the study participant is allocated to a group, the allocation is saved in a central database, and an e-mail containing the allocation is sent to the PI, the study coordinator, and to the person submitting the participant. Allocation concealment consists of four aspects: (1) requesting randomization after baseline measurement, (2) use of a random element, (3) requesting allocation for participants by two different study nurses, and (4) not disclosing full details of minimization to study nurses in accordance with the SPIRIT guideline [67]. We will publish a detailed description of the minimization scheme with the results of the trial.

It is impossible to blind participants in exercise trials. However, we prevent detection bias though objective sleep measures which are assessed by two blinded and independent assessors. Blinding is ensured by replacing the subject ID with a second unique ID number. The list matching these IDs is not accessible to the raters.

\section{Intervention and control condition}

Aerobic exercise (intervention) Patients allocated to the intervention group perform a single bout of supervised aerobic exercise. The starting time is approximately $1645 \mathrm{~h}$. The exercise mode is a bicycle ergometer (ergoselect 200, Ergoline, Bitz, Germany). After a warmup period of $5 \mathrm{~min}$, during which the intensity is gradually increased, patients maintain an intensity of $80 \%$ of the individual anaerobic threshold for $30 \mathrm{~min}$. The intensity level is chosen based on clinical experience that this corresponds to an approximate rate of perceived exertion of 13 (on a scale from 6 to 20) in this population. The duration of exercise corresponds to physical activity recommendations [181].

Control condition At the same time as individuals performing the exercise intervention, individuals allocated to the control group are placed in a room which is comparable to that of the exercise group concerning light, temperature, and absence of music.
The control group is asked to remain seated and read magazines.

The rules and schedules of the inpatient rehabilitation clinic (e.g., timing of meals, consumption of multimedia, and alcohol) limit the variability of many behavioral aspects which could influence sleep. Occasional smokers are asked to refrain from smoking after dinner on the days of polysomnographic assessment. Chronic smokers are not asked to abstain from smoking as this might be an additional stressor.

Adherence and other outcomes of interest We assess the implementation of the intervention with continuous measurement of Watts and heart rate using a Polar ${ }^{\circ} \mathrm{H} 7$ chest strap (Polar OY, Finland). We measure heart rate throughout the intervention period, including $3 \mathrm{~min}$ post-exercise. We measure perceived exertion using the Borg scale (6-20) [93]. All subjects complete a questionnaire on their current mood immediately before and at the end of the control condition as well as the exercise intervention. The Befindlichkeitsskala has adequate psychometric properties [182] and is considerably more economical than other comparable measures [183]. The questionnaire consists of 40 items on a five-point Likert scale (not at all to very much). Items load onto eight subscales (with five items each): activity (Cronbach's $\alpha=0.82$ ), elation (Cronbach's $\alpha=0.81$ ), contemplation (Cronbach's $\alpha=0.70$ ), calmness (Cronbach's $\alpha=0.78$ ), fatigue (Cronbach's $\alpha=0.88$ ), depression (Cronbach's $\alpha=0.80$ ), anger (Cronbach's $\alpha=0.86$ ), and excitement (Cronbach's $\alpha=0.73$ ) [182]. Contamination through any or additional physical activity (depending on the allocation) is assessed using a wrist-worn accelerometer (on non-dominant hand) on the days prior to and after the sleep assessments. The wrist-worn accelerometer vivofit ${ }^{\circ}$ (Garmin, Schaffhausen, Switzerland) validly assesses steps in various walking conditions [184]. Although adequate blinding cannot be achieved by design in exercise studies, this allows for a partial assessment of performance bias.

Concomitant, ancillary, and post-trial care The trial takes place in the first 5 days of the patient's psychosomatic in-patient rehabilitation. The rehabilitation programme entails different therapies, including exercise therapy. Patients included in the study are asked to refrain from exercise except as defined by the protocol on the days of testing. Patients are explicitly made aware of this aspect before enrollment. Ancillary and posttrial care is provided throughout the in-patient rehabilitation program, i.e., on average for 4 weeks after completion of the study. 
Adverse events We assess adverse events through a questionnaire. We ask patients in both groups whether they experience adverse effects on a five-point Likert scale (not at all to very) using the following categories immediately after the intervention and the following morning:

- Pain (if yes, location)

- Dizziness

- Cardiovascular symptoms (e.g., angina symptoms, cyanosis, pallor)

- Respiratory symptoms (e.g., wheezing)

- Nausea

- Falls (yes or no)

- Other (to be described)

Unplanned termination of participants and the reasons thereof (if participant proactively gives one) will also be reported.

\section{Data management Data collection}

We use castor electronic data capture software for data collection and data management [185]. This software complies with Good Clinical Practice guidelines and the European Data Protection Directive. A unique numeric subject ID is assigned to each participant to conceal the identity of participants in the database. The file which links the numeric subject ID to the participant information is kept in a password-protected folder in an encrypted digital file and on paper in a locked cabinet. These digital and paper files are exclusively stored at the study site. Only the study nurses, the study coordinator, and the principal investigator have access to this information. Regulatory agencies (i.e., ethics committee) will also be granted access upon request. Patient data are collected using electronic case report forms (eCRF). Range and dependency checks are implemented into the eCRF and completeness checks of data for each participant are performed during the trial. The eCRF can be found in Additional file 3. All study personnel have been trained in measurement procedures and data collection according to the case report form using standard operating procedures to ensure standardized data collection. Data from the polysomnography are uploaded to the CASTOR platform using the subject ID to match data.

\section{Security, storage, and access}

Study nurses, the study coordinator, the principal investigator, and monitors can access the password-protected database. The principal investigator and study coordinator define user accounts and user rights according to their responsibilities, e.g., authorization for data changes. All changes in the eCRF are saved in data trails, audit trials, and edit trails, (including reasons for changes). The data are stored for 15 years. The study coordinator and principal investigator will have full access to the trial data.

\section{Data monitoring and audits}

Due to the risk stratification of this trial, the need for a data-monitoring committee is waived. Central data monitoring is performed using the built-in modules of the CASTOR software. No interim analyses are planned. Monitoring of regulatory files, study processes, and data is conducted in four visits: before enrollment of the first patient, after enrollment of the first patient, after enrollment of $50 \%$ of patients, and after last patient last visit. Monitoring is done by the Clinical Trial Unit, Basel, Switzerland (i.e., an organization independent from the investigator and sponsor).

\section{Statistical methods Sample size calculation}

The theoretical rationale for the analysis of the primary outcome, using an ANCOVA model, is outlined above (see the "Polysomnography (baseline and follow-up)" section). Furthermore, minimization necessitates adjustment for minimization factors [186]. Sample size calculation was performed according to the procedure defined by Borm et al. [187]. The allocation ratio is 1:1. The expected treatment effect is based on the work of Passos et al. [63], who found a standardized mean difference in sleep efficiency of 0.53 . The estimate is based on this publication because it is the only one known to the authors which (1) evaluated the acute effect of (2) a similar intervention (i.e., moderate aerobic exercise) (3) in individuals with sleep disorders. Due to the well-documented ceiling and floor effects [188], meta-analyses concerning predominantly healthy individuals (explicitly excluding individuals with mental disorders) [54] are of no use. Meta-analyses including individuals with sleep complaints are limited to the analysis of the chronic effects of exercise on sleep [55, 189]. With a power of 0.8 and a two-sided alpha of $0.05,57$ subjects would be required for each group using a $t$-test. According to the method of Borm et al., this sample size can be multiplied by a 'design factor' of $\left(1-\rho^{2}\right)$, where $\rho$ is the correlation coefficient between baseline and follow-up outcome [187]. Despite contacting other researchers, the authors are not aware of any previous study which have analyzed this aspect. Hence, we need to make an estimate. Recommended values for imputation of $\rho$ vary between 0.5 (if variances are equal at pre- and post-measurements, $\rho$ is at least 0.5 ) and $0.7[190,191]$. We use a conservative estimate and let $\rho=0.5$. This leads to a design factor of $0.75\left(1-0.5^{2}=\right.$ $0.75)$. Hence, the sample size needed per group is $43(57 \times$ 
$0.75=42.75$ ). Due to the short-term nature of this study, we expect approximately half the dropout rate of trials investigating the chronic effects of exercise in patients with depression [192]. Thus, we anticipate 7\% dropouts. Therefore, the total sample size is $92(2 \times 43 \times 1.07=92)$.

\section{Analysis of the primary outcome}

The main aim of this trial is to analyze the acute effect of aerobic exercise on the subsequent night's sleep efficiency measured by polysomnography. To this end, we will compute a one-way ANCOVA with baseline sleep efficiency and minimization factors as covariates, intervention as the independent variable, and post-exercise sleep efficiency as the dependent variable. Clinical significance will be determined using the criteria defined in the American Academy of Sleep Medicine Clinical Practice guideline for the Pharmacological Treatment of Chronic Insomnia in adults. Thereby, an absolute change in sleep efficiency of $\geq 5 \%$ is deemed clinically relevant. We define responders as individuals who have an increase in sleep efficiency of $\geq 5 \%$ from baseline to post-intervention. We will report the number, the proportion, and the odds ratio of responders as well as the number needed to treat. In order to reduce attrition bias to a minimum, all analyses will follow the intention-to-treat framework.

Sensitivity analyses for the primary outcome will be performed to gauge the influence of several factors: outliers (defined as < Quartile $1-1.5 \times$ Interquartile range; $>$ Quartile $3+1.5 \times$ Interquartile range), per-protocol analysis (to reflect optimal adherence to treatment), missing data (analysis of complete data only), excluding minimization factors (age, sex, PHQ-9 score, and Pittsburgh Sleep Quality Index score), chronotypes, smoking status, and use of beta-blockers. We will report all results of sensitivity analyses. We will replace missing data using multiple imputation with the $m i$ package in $\mathrm{R}$ [193]. The quantity of missing data will be reported.

\section{Analysis of secondary outcomes}

Secondary outcomes will also be analyzed using ANCOVA models when variables have been assessed at baseline and follow-up. Hourly segments of nocturnal HRV will be analyzed using a linear mixed model with subject as random effect, adjusting for baseline and minimization factors. Acute daytime sleepiness will be assessed using repeated measures ANOVA with Benjamini-Hochberg [194] corrected post hoc paired sample $t$-tests. Measurements where only post-intervention group-comparisons are possible will be analyzed using $t$-tests. The threshold for statistical significance is set at $p<0.05$. The authors point out that the secondary analyses are not adjusted for multiple testing and are of exploratory nature.

\section{Ethics and dissemination}

Ethics approval

The Ethics Committee East Switzerland, St. Gallen, Switzerland, has approved the study (EKOS 18/089). We registered the trial with the ClinicalTrials.gov database (NCT03673397) on September 17, 2018. No protocol amendments have been made. Approval for any potential future amendments will be obtained from the local ethics committee.

\section{Dissemination policy}

We will disseminate trial results to all stakeholders, e.g., participants (if they choose to receive these at enrollment), physicians, and study nurses. Results will be published in a peer-reviewed journal and presented at conferences as well as invited talks. Authorship on peerreviewed publications will be based on contributions toward design, fundraising, data collection, analysis, and manuscript preparation. De-identified (i.e., coded) individual participant data that underlie the results of published articles, including data dictionaries, will be available upon request under the creative commons license CC-BY. Requests will only be granted for use in individual participant data meta-analysis which has been approved by an independent review committee. Exceptions to these rules are reserved within the context of peer-reviewed publications, provided that data integrity remains intact. Data will be provided upon request immediately after publication of peer-reviewed articles with no end date. Requests should be sent to the e-mail address detailed in the following Dataverse repository (https://doi.org/10.7910/DVN/WASN36).

\section{Discussion}

The goal of this two-arm parallel group, individually randomized, single-blind, controlled trial is to assess the superiority of an acute bout of aerobic exercise compared to no intervention in improving the subsequent night's sleep efficiency in patients with a primary diagnosis of depression. To the knowledge of the authors, this is the first trial to investigate the acute effects of exercise on sleep in this population.

The main strength of this study is the objective measurement of sleep. Moreover, multiple secondary outcomes were carefully selected to provide clinicians, patients, and policy makers with a comprehensive picture of the effects this intervention may have. Explicit inclusion of patients with comorbidities should enhance the external validity of this study. Accordingly, we provide extensive baseline characterization.

The main limitation of this trial is the restricted polysomnographic EEG montage. More detailed analyses, such as spectral EEG analysis, will therefore not be possible. Extensive discussions in our research group 
resulted in an explicit trade-off between the patients' discomfort (number of EEG channels) and feasibility of recruitment. The restricted EEG montage will help to recruit the necessary number of patients in an adequate period in this clinical setting. The pre-selection of patients who are treated in this rehabilitation clinic might pose a further limitation. Extensive baseline characterization will help readers identify limits to external validity. Patients cannot be blinded against allocation in exercise trials. We try to overcome this limitation through various measures (see above).

There is compelling evidence for the effectiveness of various therapies for both insomnia and depression. Nevertheless, many of these therapies have shortcomings. Development of further therapeutic options, which can be administered in addition to these therapies, are therefore needed. Current literature suggests that acute aerobic exercise might improve both depression and insomnia. Although chronic aerobic exercise is included in sleep hygiene recommendations, there is uncertainty concerning the acute effects of aerobic exercise on sleep in patients with depression. This trial aims to close this gap in the literature as well as to help the increasing number of patients with depression.

\section{Trial status}

We registered this trial on September 17, 2018, in the ClinicalTrials.gov database (NCT03673397). All items of the World Health Organization Trial Registration Data Set can be found in the ClinicalTrials.gov registration. This is the first version of the protocol, i.e., we have made no amendments. Recruitment began on September 24, 2018. Recruitment is expected to be completed by October 2019.

\section{Additional files}

\author{
Additional file 1: SPIRIT checklist. (PDF $171 \mathrm{~kb}$ ) \\ Additional file 2: Original consent form in German. (PDF 561 kb) \\ Additional file 3: Electronic case report form (eCRF). (PDF 504 kb)
}

\footnotetext{
Abbreviations

ANCOVA: Analysis of covariance; AR: Autoregressive modeling; BMI: Body mass index; BP: Blood pressure; CBT-I: Cognitive behavioral therapy for insomnia; ECG: Electrocardiogram; eCRF: Electronic case report form; EEG: Electroencephalogram; EOG: Electrooculogram; FFT: Fast Fourier transform; HF: High frequency power; HRV: Heart rate variability; ICD10: International Statistical Classification of Diseases and Related Health Problems, 10th revision; LF: Low frequency power; LSP: Lomb-Scargle periodogram; N1-N3: Stage 1-3 sleep; NA: Number of awakenings; ODI: Oxygen desaturation index; PHQ: Patient Health Questionnaire; REM: Rapid eye movement; SE: Sleep efficiency; SOL: Sleep onset latency; SPIRIT: Standard Protocol Items: Recommendations for Interventional Trials (SPIRIT); TRT: Total recording time; TST: Total sleep time; WASO: Wake after sleep onset
}

\section{Publisher's Note}

Springer Nature remains neutral with regard to jurisdictional claims in published maps and institutional affiliations.

\section{Acknowledgements}

Not applicable.

\section{Authors' contributions}

$\mathrm{GB}, \mathrm{DS}, \mathrm{HP}, \mathrm{TZS}, \mathrm{MG}, \mathrm{RvK}$, and AST contributed to the design, revised the manuscript, and approved the final manuscript. GB conceived the trial design, drafted the manuscript, registered the protocol with ClinicalTrials.gov, and managed the overall project.

\section{Funding}

The majority of work was funded through an industry sponsored $\mathrm{PhD}$, provided by OBERWAID AG, St. Gallen, Switzerland.

\section{Availability of data and materials}

The dataset supporting the conclusions of this article is available in the Dataverse repository (https://doi.org/10.7910/DVN/WASN36). De-identified (i.e., coded) individual participant data that underlie the results of published articles, including data dictionaries, will be available upon request under the creative commons license CC-BY. Requests will only be granted for use in individual participant data meta-analysis which has been approved by an independent review committee. Exceptions to these rules are reserved within the context of peer-reviewed publications, provided that data integrity remains intact. Data will be provided upon request immediately after publication of peer-reviewed articles with no end date. Requests should be sent to the e-mail address detailed in the Dataverse repository mentioned above.

Ethics approval and consent to participate

The Ethics Committee East Switzerland, St. Gallen, Switzerland, has approved the study (EKOS 18/089).

Consent for publication Not applicable.

\section{Competing interests}

Gavin Brupbacher is funded through an industry sponsored PhD, provided by OBERWAID AG, St. Gallen, Switzerland. Dr. Doris Straus and Dr. Hildburg Porschke are employed by OBERWAID AG. Arno Schmidt-Trucksäss and Roland von Känel are on the scientific advisory board of the Oberwaid AG. All other authors declare no competing interests.

\section{Author details}

${ }^{1}$ Division of Sports and Exercise Medicine, Department of Sport, Exercise and Health, University of Basel, Birsstrasse 320 B, 4052 Basel, Switzerland. ${ }^{2}$ OBERWAID AG, Rorschacher Strasse 311, 9016 St. Gallen, Switzerland. ${ }^{3}$ Department of Psychology, Division of Clinical Psychology and Epidemiology, University of Basel, Basel, Switzerland. ${ }^{4}$ Division of Sport and Psychosocial Health, Department of Sport, Exercise and Health, University of Basel, Birsstrasse 320 B, 4052 Basel, Switzerland. ${ }^{5}$ Department of Consultation-Liaison Psychiatry and Psychosomatic Medicine, University Hospital Zurich, Culmannstrasse 8, 8091 Zurich, Switzerland.

Received: 30 November 2018 Accepted: 8 May 2019

Published online: 13 June 2019

References

1. Steel Z, Marnane C, Iranpour C, Chey T, Jackson JW, Patel V, et al. The global prevalence of common mental disorders: a systematic review and metaanalysis 1980-2013. Int J Epidemiol. 2014;43:476-93.

2. Kessler RC, Aguilar-Gaxiola S, Alonso J, Chatteriji S, Lee S, Ormel J, et al. The global burden of mental disorders: an update from the WHO World Mental Health (WMH) surveys. Epidemiol Psichiatr Soc. 2009;18:23-33.

3. Goodell S, Druss BG, Walker ER. Mental disorders and medical comorbidity. Princeton: Robert Wood Johnson Foundation; 2011. https://www. researchgate.net/profile/Elizabeth_Walker12/publication/51220912_Mental_ Disorders_and_Medical_Comorbidity/links/0a85e53c973139cf16000000/ Mental-Disorders-and-Medical-Comorbidity.pdf 
4. Gan Y, Gong Y, Tong X, Sun H, Cong Y, Dong X, et al. Depression and the risk of coronary heart disease: a meta-analysis of prospective cohort studies. BMC Psychiatry. 2014;14. https://doi.org/10.1186/s12888-014-0371-z.

5. Vancampfort D, Rosenbaum S, Schuch F, Ward PB, Richards J, Mugisha J, et al. Cardiorespiratory fitness in severe mental illness: a systematic review and meta-analysis. Sports Med. 2017:47:343-52.

6. Kodama S, Saito K, Tanaka S, Maki M, Yachi Y, Asumi M, et al. Cardiorespiratory fitness as a quantitative predictor of all-cause mortality and cardiovascular events in healthy men and women: a meta-analysis. JAMA. 2009;301:2024-35.

7. National Institute for Health and Clinical Excellence. Depression in adults: recognition and management. 2016. https://www.nice.org.uk/guidance/ cg90. Accessed 16 Jan 2018.

8. Holsboer-Trachsler E, Hättenschwiler J, Beck J, Brand S, Hemmeter U, Keck $\mathrm{ME}$, et al. Die somatische Behandlung der unipolaren depressiven Störungen. Swiss Med Forum. 2010;10:802-9.

9. Cuijpers P, Sijbrandij M, Koole SL, Andersson G, Beekman AT, Reynolds CF. Adding psychotherapy to antidepressant medication in depression and anxiety disorders: a meta-analysis. World Psychiatry. 2014;13:56-67.

10. Rush AJ, Trivedi MH, Wisniewski SR, Nierenberg AA, Stewart JW, Warden $D$, et al. Acute and longer-term outcomes in depressed outpatients requiring one or several treatment steps: a STAR*D report. Am J Psychiatry. 2006;163:1905-17.

11. Omvik S, Pallesen S, Bjorvatn B, Sivertsen B, Havik OE, Nordhus IH. Patient characteristics and predictors of sleep medication use. Int Clin Psychopharmacol. 2010;25:91-100.

12. Cheung JMY, Bartlett DJ, Armour CL, Saini B, Laba T-L. Patient preferences for managing insomnia: a discrete choice experiment. The Patient. 2018;11:503-14.

13. McHugh RK, Whitton SW, Peckham AD, Welge JA, Otto MW. Patient preference for psychological vs pharmacologic treatment of psychiatric disorders: a meta-analytic review. J Clin Psychiatry. 2013;74:595-602.

14. Edinger JD, Bonnet MH, Bootzin RR, Doghramji K, Dorsey CM, Espie CA, et al. Derivation of research diagnostic criteria for insomnia: report of an American Academy of Sleep Medicine Work Group. Sleep. 2004;27:1567-96.

15. Baglioni C, Spiegelhalder K, Lombardo C, Riemann D. Sleep and emotions: a focus on insomnia. Sleep Med Rev. 2010;14:227-38.

16. Stepanski EJ, Rybarczyk B. Emerging research on the treatment and etiology of secondary or comorbid insomnia. Sleep Med Rev. 2006;10:7-18.

17. Medalie L, Cifu AS. Management of Chronic Insomnia Disorder in Adults. JAMA. 2017;317:762-3.

18. Schutte-Rodin S, Broch L, Buysse D, Dorsey C, Sateia M. Clinical guideline for the evaluation and management of chronic insomnia in adults. J Clin Sleep Med. 2008;4:487-504.

19. Wu JQ, Appleman ER, Salazar RD, Ong JC. Cognitive behavioral therapy for insomnia comorbid with psychiatric and medical conditions: a metaanalysis. JAMA Intern Med. 2015;175:1461-72.

20. Buscemi N, Vandermeer B, Friesen C, Bialy L, Tubman M, Ospina M, et al. The efficacy and safety of drug treatments for chronic insomnia in adults: a meta-analysis of RCTs. J Gen Intern Med. 2007;22:1335-50.

21. Huedo-Medina TB, Kirsch I, Middlemass J, Klonizakis M, Siriwardena AN. Effectiveness of non-benzodiazepine hypnotics in treatment of adult insomnia: meta-analysis of data submitted to the Food and Drug Administration. BMJ. 2012;345:e8343.

22. Kurko TAT, Saastamoinen LK, Tähkäpää S, Tuulio-Henriksson A, Taiminen T, Tiihonen J, et al. Long-term use of benzodiazepines: Definitions, prevalence and usage patterns - a systematic review of register-based studies. Eur Psychiatry. 2015;30:1037-47.

23. Sakshaug S, Handal M, Hjellvik V, Berg C, Ripel Å, Gustavsen I, et al. Longterm use of Z-hypnotics and co-medication with benzodiazepines and opioids. Basic Clin Pharmacol Toxicol. 2017;120:292-8.

24. Pillai V, Cheng P, Kalmbach DA, Roehrs T, Roth T, Drake CL. Prevalence and predictors of prescription sleep aid use among individuals with DSM-5 insomnia: the role of hyperarousal. Sleep. 2016;39:825-32

25. Pillai V, Roth T, Roehrs T, Moss K, Peterson EL, Drake CL. Effectiveness of benzodiazepine receptor agonists in the treatment of insomnia: an examination of response and remission rates. Sleep. 2017:40.

26. Gunja N. In the Zzz Zone: The effects of Z-drugs on human performance and driving. J Med Toxicol. 2013;9:163-71.

27. Kripke DF. Hypnotic drug risks of mortality, infection, depression, and cancer: but lack of benefit. F1000Research. 2016;5:918.
28. Lai M-M, Lin C-C, Lin C-C, Liu C-S, Li T-C, Kao C-H. Long-term use of zolpidem increases the risk of major injury: a population-based cohort study. Mayo Clin Proc. 2014;89:589-94.

29. Morin CM, Gaulier B, Barry T, Kowatch RA. Patients' acceptance of psychological and pharmacological therapies for insomnia. Sleep. 1992;15:302-5.

30. Vincent $\mathrm{N}$, Lionberg $\mathrm{C}$. Treatment preference and patient satisfaction in chronic insomnia. Sleep. 2001;24:411-7.

31. van MJG, Hoogendijk WJG, Vogelzangs N, van DR, Penninx BWJH. Insomnia and sleep duration in a large cohort of patients with major depressive disorder and anxiety disorders. J Clin Psychiatry. 2010;71:239-46.

32. Spiegelhalder K, Regen W, Nanovska S, Baglioni C, Riemann D. Comorbid sleep disorders in neuropsychiatric disorders across the life cycle. Curr Psychiatry Rep. 2013;15:364.

33. Li L, Wu C, Gan Y, Qu X, Lu Z. Insomnia and the risk of depression: a metaanalysis of prospective cohort studies. BMC Psychiatry. 2016;16:375.

34. Alvaro PK, Roberts RM, Harris JK. A systematic review assessing bidirectionality between sleep disturbances, anxiety, and depression. Sleep. 2013;36:1059-68.

35. Jansson-Fröjmark M, Lindblom K. A bidirectional relationship between anxiety and depression, and insomnia? A prospective study in the general population. J Psychosom Res. 2008;64:443-9.

36. Sivertsen B, Salo P, Mykletun A, Hysing M, Pallesen S, Krokstad S, et al. The bidirectional association between depression and insomnia: the HUNT study. Psychosom Med. 2012;74:758-65.

37. Franzen PL, Buysse DJ. Sleep disturbances and depression: risk relationships for subsequent depression and therapeutic implications. Dialogues Clin Neurosci. 2008;10:473-81.

38. Carney CE, Segal ZV, Edinger JD, Krystal AD. A comparison of rates of residual insomnia symptoms following pharmacotherapy or cognitivebehavioral therapy for major depressive disorder. J Clin Psychiatry. 2007;68:254-60.

39. Nierenberg AA, Husain MM, Trivedi MH, Fava M, Warden D, Wisniewski $\mathrm{SR}$, et al. Residual symptoms after remission of major depressive disorder with citalopram and risk of relapse: a STAR* $D$ report. Psychol Med. 2010;40:41-50.

40. Krystal AD. Psychiatric disorders and sleep. Neurol Clin. 2012;30:1389-413.

41. Sakurai H, Suzuki T, Yoshimura K, Mimura M, Uchida H. Predicting relapse with individual residual symptoms in major depressive disorder: a reanalysis of the STAR*D data. Psychopharmacology. 2017;234:2453-61.

42. Bernert RA, Kim JS, Iwata NG, Perlis ML. Sleep disturbances as an evidencebased suicide risk factor. Curr Psychiatry Rep. 2015;17:554.

43. Winsper C, Tang NKY. Linkages between insomnia and suicidality: prospective associations, high-risk subgroups and possible psychological mechanisms. Int Rev Psychiatry Abingdon Engl. 2014; 26:189-204.

44. He Q, Zhang P, Li G, Dai H, Shi J. The association between insomnia symptoms and risk of cardio-cerebral vascular events: A meta-analysis of prospective cohort studies. Eur J Prev Cardiol. 2017;24:1071-82.

45. Kessler RC, Berglund PA, Coulouvrat C, Hajak G, Roth T, Shahly V, et al. Insomnia and the performance of US workers: results from the america insomnia survey. Sleep. 2011:34:1161-71.

46. Espie CA, Pawlecki B, Waterfield D, Fitton K, Radocchia M, Luik Al. Insomnia symptoms and their association with workplace productivity: cross-sectional and pre-post intervention analyses from a large multinational manufacturing company. Sleep Health. 2018;4:307-12.

47. Reynolds AC, Appleton SL, Gill TK, Taylor AW, McEvoy RD, Ferguson SA, et al. Sickness absenteeism is associated with sleep problems independent of sleep disorders: results of the 2016 Sleep Health Foundation national survey. Sleep Health. 2017:3:357-61.

48. Kanerva N, Pietiläinen O, Lallukka T, Rahkonen O, Lahti J. Unhealthy lifestyle and sleep problems as risk factors for increased direct employers' cost of short-term sickness absence. Scand J Work Environ Health. 2018:44(2):192-201.

49. Uehli K, Mehta AJ, Miedinger D, Hug K, Schindler C, Holsboer-Trachsler E, et al. Sleep problems and work injuries: A systematic review and meta-analysis. Sleep Med Rev. 2014;18:61-73.

50. Dong L, Agnew J, Mojtabai R, Surkan PJ, Spira AP. Insomnia as a predictor of job exit among middle-aged and older adults: results from the Health and Retirement Study. J Epidemiol Community Health. 2017:71(8):750-757.

51. Paunio T, Korhonen T, Hublin C, Partinen M, Koskenvuo K, Koskenvuo M, et al. Poor sleep predicts symptoms of depression and disability retirement due to depression. J Affect Disord. 2015;172:381-9. 
52. NIH State-of-the-Science Conference Statement on manifestations and management of chronic insomnia in adults. NIH Consens State Sci Statements. 2005;22:1-30.

53. Lang C, Kalak N, Brand S, Holsboer-Trachsler E, Pühse U, Gerber M. The relationship between physical activity and sleep from mid adolescence to early adulthood. A systematic review of methodological approaches and meta-analysis. Sleep Med Rev. 2015;28:28-41.

54. Kredlow MA, Capozzoli MC, Hearon BA, Calkins AW, Otto MW. The effects of physical activity on sleep: a meta-analytic review. J Behav Med. 2015;38:427-49.

55. Yang $\mathrm{P}-\mathrm{Y}, \mathrm{Ho} \mathrm{K}-\mathrm{H}$, Chen $\mathrm{H}-\mathrm{C}$, Chien M-Y. Exercise training improves sleep quality in middle-aged and older adults with sleep problems: a systematic review. J Physiother. 2012;58:157-63.

56. Passos GS, Poyares DLR, Santana MG, Tufik S, de Mello MT. Is exercise an alternative treatment for chronic insomnia? Clinics. 2012;67:653-60.

57. Cooney GM, Dwan K, Greig CA, Lawlor DA, Rimer J, Waugh FR, et al. Exercise for depression. Cochrane Database Syst Rev. 2013;12(9):CD004366

58. Stubbs B, Rosenbaum S, Vancampfort D, Ward PB, Schuch FB. Exercise improves cardiorespiratory fitness in people with depression: A metaanalysis of randomized control trials. J Affect Disord. 2016;190:249-53.

59. American Sleep Association. Sleep hygiene tips. 2017. https://www. sleepassociation.org/patients-general-public/insomnia/sleep-hygiene-tips/. Accessed 28 Apr 2017

60. Gold PW. The organization of the stress system and its dysregulation in depressive illness. Mol Psychiatry. 2015;20:32-47.

61. Myllymäki T, Kyröläinen $H$, Savolainen $K$, Hokka L, Jakonen $R$, Juuti $T$, et al. Effects of vigorous late-night exercise on sleep quality and cardiac autonomic activity. J Sleep Res. 2011;20(1 Pt 2):146-53.

62. Myllymäki T, Rusko H, Syväoja H, Juuti T, Kinnunen M-L, Kyröläinen H. Effects of exercise intensity and duration on nocturnal heart rate variability and sleep quality. Eur J Appl Physiol. 2012;112:801-9.

63. Passos GS, Poyares D, Santana MG, Garbuio SA, Tufik S, Mello MT. Effect of acute physical exercise on patients with chronic primary insomnia. J Clin Sleep Med. 2010;6:270-5.

64. Banno M, Harada Y, Taniguchi M, Tobita R, Tsujimoto H, Tsujimoto Y, et al. Exercise can improve sleep quality: a systematic review and meta-analysis. PeerJ. 2018;6:e5172.

65. Baron KG, Reid KJ, Zee PC. Exercise to improve sleep in insomnia: exploration of the bidirectional effects. J Clin Sleep Med. 2013;9:819-24.

66. Miura A, Myouken S, Yamada M, Fujihara C, Miura K, Kashima H, et al. Effects of aerobic exercise in early evening on the following nocturnal sleep and its haemodynamic response. Res Sports Med Print. 2016;24:16-29.

67. Chan A-W, Tetzlaff JM, Altman DG, Laupacis A, Gøtzsche PC, Krleža-Jerić K, et al. SPIRIT 2013 statement: defining standard protocol items for clinical trials. Ann Intern Med. 2013;158:200-7.

68. Klerman EB, Wang W, Duffy JF, Dijk D-J, Czeisler CA, Kronauer RE. Survival analysis indicates that age-related decline in sleep continuity occurs exclusively during NREM sleep. Neurobiol Aging. 2013;34:309-18.

69. American College of Sports Medicine. ACSM's Guidelines for Exercise Testing and Prescription. 8th ed. Philadelphia: LWW; 2009.

70. Stoschitzky K, Sakotnik A, Lercher P, Zweiker R, Maier R, Liebmann P, et al. Influence of beta-blockers on melatonin release. Eur J Clin Pharmacol. 1999;55:111-5.

71. Stoschitzky K, Stoschitzky G, Brussee H, Bonelli C, Dobnig H. Comparing beta-blocking effects of bisoprolol, carvedilol and nebivolol. Cardiology. 2006;106:199-206.

72. Brismar K, Mogensen L, Wetterberg L. Depressed melatonin secretion in patients with nightmares due to beta-adrenoceptor blocking drugs. Acta Med Scand. 1987;221:155-8.

73. Demitrack MA, Lewy AJ, Reus VI. Pineal-adrenal interactions: the effect of acute pharmacological blockade of nocturnal melatonin secretion. Psychiatry Res. 1990;32:183-9.

74. Dimsdale JE, Norman D, DeJardin D, Wallace MS. The effect of opioids on sleep architecture. J Clin Sleep Med. 2007;3:33-6.

75. Matos G, Tufik S, Scorza FA, Cavalheiro EA, Andersen ML. Sleep and epilepsy: exploring an intriguing relationship with a translational approach. Epilepsy Behav. 2013;26:405-9.

76. Stiasny-Kolster K, Möller JC, Heinzel-Gutenbrunner M, Baum E, Ries V, Oertel $\mathrm{WH}$. Validation of the restless legs syndrome screening questionnaire (RLSSQ). Somnologie Schlafforschung Schlafmed. 2009;13:37-42.
77. Bogan RK. Effects of restless legs syndrome (RLS) on sleep. Neuropsychiatr Dis Treat. 2006;2:513-9.

78. Chung F, Liao P, Elsaid H, Islam S, Shapiro CM, Sun Y. Oxygen desaturation index from nocturnal oximetry: a sensitive and specific tool to detect sleep-disordered breathing in surgical patients. Anesth Analg. 2012;114:993-1000.

79. Hang L-W, Wang H-L, Chen J-H, Hsu J-C, Lin H-H, Chung W-S, et al. Validation of overnight oximetry to diagnose patients with moderate to severe obstructive sleep apnea. BMC Pulm Med. 2015;15. https://doi. org/10.1186/s12890-015-0017-Z.

80. Fabius TM, Benistant JR, Bekkedam L, van der Palen J, de Jongh FHC, Eijsvogel MMM. Validation of the oxygen desaturation index in the diagnostic workup of obstructive sleep apnea. Sleep Breath. 2018;23(1): 57-63.

81. Morin CM. Measuring outcomes in randomized clinical trials of insomnia treatments. Sleep Med Rev. 2003;7:263-79.

82. Baglioni C, Nanovska S, Regen W, Spiegelhalder K, Feige B, Nissen C, et al. Sleep and mental disorders: A meta-analysis of polysomnographic research. Psychol Bull. 2016;142:969-90.

83. Palagini L, Baglioni C, Ciapparelli A, Gemignani A, Riemann D. REM sleep dysregulation in depression: State of the art. Sleep Med Rev. 2013;17:377-90.

84. Kaplan KA, Hirshman J, Hernandez B, Stefanick ML, Hoffman AR, Redline S, et al. When a gold standard isn't so golden: Lack of prediction of subjective sleep quality from sleep polysomnography. Biol Psychol. 2017;123:37-46.

85. Herbert V, Pratt D, Emsley R, Kyle SD. Predictors of nightly subjectiveobjective sleep discrepancy in poor sleepers over a seven-day period. Brain Sci. 2017;7.

86. Cheung JMY, Bartlett DJ, Armour CL, Saini B. Treating Insomnia: a review of patient perceptions toward treatment. Behav Sleep Med. 2016;14:235-66.

87. Sirdifield C, Chipchase SY, Owen S, Siriwardena AN. A systematic review and meta-synthesis of patients' experiences and perceptions of seeking and using benzodiazepines and Z-drugs: towards safer prescribing. The Patient. 2017;10:1-15.

88. Sateia MJ, Buysse DJ, Krystal AD, Neubauer DN, Heald JL. Clinical practice guideline for the pharmacologic treatment of chronic insomnia in adults: an American Academy of Sleep Medicine Clinical Practice Guideline. J Clin Sleep Med. 2017;13:307-49.

89. Bentley DJ, Newell J, Bishop D. Incremental exercise test design and analysis. Sports Med. 2007;37:575-86.

90. Bonaventura JM, Sharpe K, Knight E, Fuller KL, Tanner RK, Gore CJ. Reliability and accuracy of six hand-held blood lactate analysers. J Sports Sci Med. 2015;14:203-14.

91. Caminal P, Sola F, Gomis P, Guasch E, Perera A, Soriano N, et al. Validity of the Polar V800 monitor for measuring heart rate variability in mountain running route conditions. Eur J Appl Physiol. 2018;118:669-77.

92. Yamamoto $Y$, Hughson RL, Peterson JC. Autonomic control of heart rate during exercise studied by heart rate variability spectral analysis. J Appl Physiol. 1991;71:1136-42.

93. Borg G. Anstrengungsempfinden und körperliche Aktivität. Dtsch Ärztebl. 2004;101:A1016-21.

94. Dickhuth H-H, Huonker M, Münzel T, Drexler H, Berg A, Keul J. Individual anaerobic threshold for evaluation of competitive athletes and patients with left ventricular dysfunction. In: Advances in Ergometry. Edited by Bachl N, Graham, T, Löllgen, H. Berlin, Heidelberg: Springer; 1991. p. 173-9. https://doi.org/10.1007/978-3642-76442-4_26

95. Obbarius A, van Maasakkers L, Baer L, Clark DM, Crocker AG, de Beurs E, et al. Standardization of health outcomes assessment for depression and anxiety: recommendations from the ICHOM Depression and Anxiety Working Group. Qual Life Res. 2017;26:3211-25.

96. Kroenke K, Spitzer RL, Williams JB. The PHQ-9: validity of a brief depression severity measure. J Gen Intern Med. 2001;16:606-13.

97. Zigmond AS, Snaith RP. The hospital anxiety and depression scale. Acta Psychiatr Scand. 1983;67:361-70.

98. Bjelland I, Dahl AA, Haug TT, Neckelmann D. The validity of the Hospital Anxiety and Depression Scale. An updated literature review. J Psychosom Res. 2002:52:69-77.

99. Brennan C, Worrall-Davies A, McMillan D, Gilbody S, House A. The Hospital Anxiety and Depression Scale: a diagnostic meta-analysis of case-finding ability. J Psychosom Res. 2010;69:371-8. 
100. Norton S, Cosco T, Doyle F, Done J, Sacker A. The Hospital Anxiety and Depression Scale: A meta confirmatory factor analysis. J Psychosom Res. 2013;74:74-81.

101. Herrmann C, Buss U, HADS-D SRP. Hospital Anxiety and Depression Scale Deutsche Version (HADS-D - Hospital Anxiety and Depression Scale German version). Bern: Huber; 2011.

102. Klein EM, Brähler E, Dreier M, Reinecke L, Müller KW, Schmutzer G, et al. The German version of the Perceived Stress Scale - psychometric characteristics in a representative German community sample. BMC Psychiatry. 2016;16. https://doi.org/10.1186/s12888-016-0875-9.

103. Kroenke K, Spitzer RL, Williams JB. The PHQ-15: validity of a new measure for evaluating the severity of somatic symptoms. Psychosom Med. 2002;64:258-66.

104. Linn BS, Linn M, Gurel L. Cumulative illness rating scale. J Am Geriatr Soc. 1968;16:622-6.

105. Backhaus J, Riemann D. Schlafstörungen bewältigen [Coping with sleep disorders]. Weinheim: Beltz Psychology Verlags Union; 1996.

106. Buysse DJ, Reynolds CF, Monk TH, Berman SR, Kupfer DJ. The Pittsburgh Sleep Quality Index: a new instrument for psychiatric practice and research. Psychiatry Res. 1989;28:193-213.

107. Chiu H-Y, Chang L-Y, Hsieh Y-J, Tsai P-S. A meta-analysis of diagnostic accuracy of three screening tools for insomnia. J Psychosom Res. 2016;87: 85-92.

108. Drake C, Richardson G, Roehrs T, Scofield H, Roth T. Vulnerability to stressrelated sleep disturbance and hyperarousal. Sleep. 2004;27:285-91.

109. Kalmbach DA, Pillai V, Arnedt JT, Drake CL. Identifying at-risk individuals for insomnia using the Ford Insomnia Response to Stress Test. Sleep. 2016;39: 449-56.

110. Dieck A, Helbig S, Drake CL, Backhaus J. Validation of the German version of the Ford Insomnia Response to Stress Test. J Sleep Res. 108;27(3):e12621.

111. Harvey AG, Tang NKY, Browning L. Cognitive approaches to insomnia. Clin Psychol Rev. 2005;25:593-611.

112. Lang C, Brand S, Holsboer-Trachsler E, Pühse U, Colledge F, Gerber M. Validation of the German version of the short form of the dysfunctional beliefs and attitudes about sleep scale (DBAS-16). Neurol Sci. 2017;38(6): 1047-1058.

113. Ishak WW, Bagot K, Thomas S, Magakian N, Bedwani D, Larson D, et al. Quality of life in patients suffering from insomnia. Innov Clin Neurosci. 2012; 9:13-26.

114. Hein $M$, Lanquart J-P, Loas G, Hubain P, Linkowski P. Prevalence and risk factors of excessive daytime sleepiness in insomnia sufferers: A study with 1311 individuals. J Psychosom Res. 2017;103:63-9.

115. Slater G, Steier J. Excessive daytime sleepiness in sleep disorders. J Thorac Dis. 2012:4:608-16.

116. Bloch KE, Schoch OD, Zhang JN, Russi EW. German version of the Epworth Sleepiness Scale. Respir Int Rev Thorac Dis. 1999;66:440-7.

117. Chan JWY, Lam SP, Li SX, Yu MWM, Chan NY, Zhang J, et al. Eveningness and insomnia: independent risk factors of nonremission in major depressive disorder. Sleep. 2014;37:911-7.

118. Horne JA, Ostberg O. A self-assessment questionnaire to determine morningness-eveningness in human circadian rhythms. Int J Chronobiol. 1976;4:97-110.

119. Kantermann $T$, Sung $H$, Burgess HJ. Comparing the MorningnessEveningness Questionnaire and Munich ChronoType Questionnaire to the dim light melatonin onset. J Biol Rhythm. 2015:30:449-53.

120. Griefahn B, Künemund C, Bröde P, Mehnert P. Zur Validität der deutschen Übersetzung des Morningness-Eveningness-Questionnaires von Horne und Östberg. Somnologie. 2001;5:71-80.

121. Water ATMVD, Holmes A, Hurley DA. Objective measurements of sleep for non-laboratory settings as alternatives to polysomnography - a systematic review. J Sleep Res. 2011;20:183-200.

122. Newell J, Mairesse $O$, Verbanck $P$, Neu D. Is a one-night stay in the lab really enough to conclude? First-night effect and night-to-night variability in polysomnographic recordings among different clinical population samples. Psychiatry Res. 2012;200:795-801.

123. Song S, Geng Z, Zhai S, Xu J, Hou G, Zhang X. Changes of sleep adaptation in hospitalized patients with depression. Cell Biochem Biophys. 2013;66:347-50.

124. Mendels J, Hawkins DR. Sleep laboratory adaptation in normal subjects and depressed patients ("first night effect"). Electroencephalogr Clin Neurophysiol. 1967;22:556-8.
125. Toussaint M, Luthringer R, Schaltenbrand N, Carelli G, Lainey E, Jacqmin A, et al. First-night effect in normal subjects and psychiatric inpatients. Sleep. 1995;18:463-9.

126. Bland JM, Altman DG. Best (but oft forgotten) practices: testing for treatment effects in randomized trials by separate analyses of changes from baseline in each group is a misleading approach. Am J Clin Nutr. 2015;102:991-4.

127. Vickers AJ, Altman DG. Analysing controlled trials with baseline and follow up measurements. BMJ. 2001;323:1123-4.

128. Berry RB, Brooks R, Gamaldo CE, Harding SM, Marcus CL, Vaughn BV. The AASM manual for the scoring of sleep and associated events. Rules Terminol Tech Specif Darien III Am Acad Sleep Med. 2012. http://www.aasmnet.org/resources/pdf/scoring-manual-preface.pdf. Accessed 5 May 2017.

129. Fietze I, Penzel T, Partinen M, Sauter J, Küchler G, Suvoro A, et al. Actigraphy combined with EEG compared to polysomnography in sleep apnea patients. Physiol Meas. 2015;36:385-96.

130. Brand S, Kalak N, Gerber M, Kirov R, Pühse U, Holsboer-Trachsler E. High selfperceived exercise exertion before bedtime is associated with greater objectively assessed sleep efficiency. Sleep Med. 2014. https://doi.org/10. 1016/j.sleep.2014.05.016

131. Cohen BE, Edmondson D, Kronish IM. State of the art review: Depression, stress, anxiety, and cardiovascular disease. Am J Hypertens. 2015;28:1295-302.

132. Penninx BWJH. Depression and cardiovascular disease: Epidemiological evidence on their linking mechanisms. Neurosci Biobehav Rev. 2017; 74, Part B:277-86.

133. Javaheri S, Redline S. Insomnia and risk of cardiovascular disease. Chest. 2017;152(2):435-444

134. Yano Y, Kario K. Nocturnal blood pressure and cardiovascular disease: a review of recent advances. Hypertens Res. 2012;35:695-701.

135. Task Force of the European Society of Cardiology the North American Society of Pacing. Heart Rate Variability Standards of Measurement, Physiological Interpretation, and Clinical Use. Circulation. 1996;93:1043-65.

136. Lerma C, Martinez A, Ruiz N, Vargas A, Infante O, Martinez-Lavin M. Nocturnal heart rate variability parameters as potential fibromyalgia biomarker: correlation with symptoms severity. Arthritis Res Ther. 2011; 13:R185.

137. Hynynen E, Vesterinen V, Rusko H, Nummela A. Effects of moderate and heavy endurance exercise on nocturnal HRV. Int J Sports Med. 2010;31: 428-32.

138. Boudreau P, Yeh W-H, Dumont GA, Boivin DB. Circadian variation of heart rate variability across sleep stages. Sleep. 2013;36:1919-28.

139. Johann AF, Hertenstein E, Kyle SD, Baglioni C, Feige B, Nissen C, et al. Insomnia with objective short sleep duration is associated with longer duration of insomnia in the Freiburg Insomnia Cohort compared to insomnia with normal sleep duration, but not with hypertension. PLoS One. 2017;12:e0180339.

140. Vgontzas AN, Fernandez-Mendoza J, Liao D, Bixler EO. Insomnia with objective short sleep duration: the most biologically severe phenotype of the disorder. Sleep Med Rev. 2013;17:241-54.

141. Tobaldini E, Nobili L, Strada S, Casali KR, Braghiroli A, Montano N. Heart rate variability in normal and pathological sleep. Front Physiol. 2013;4:294.

142. Burr RL. Interpretation of normalized spectral heart rate variability indices in sleep research: a critical review. Sleep. 2007;30:913-9.

143. Laborde S, Mosley E, Thayer JF. Heart rate variability and cardiac vagal tone in psychophysiological research - recommendations for experiment planning, data analysis, and data reporting. Front Psychol. 2017;8. https:// doi.org/10.3389/fpsyg.2017.00213.

144. Heathers JAJ. Everything Hertz: methodological issues in short-term frequency-domain HRV. Front Physiol. 2014;5:177.

145. Quintana DS, Alvares GA. Heathers JA. Guidelines for reporting articles on psychiatry and heart rate variability (GRAPH): recommendations to advance research communication. Transl Psychiatry. 2016;6:e803.

146. Lomb NR. Least-squares frequency analysis of unequally spaced data. Astrophys Space Sci. 1976;39:447-62.

147. Scargle JD. Studies in astronomical time series analysis. II-Statistical aspects of spectral analysis of unevenly spaced data. Astrophys J. 1982;263:835-53.

148. Laguna P, Moody GB, Mark RG. Power spectral density of unevenly sampled data by least-square analysis: performance and application to heart rate signals. IEEE Trans Biomed Eng. 1998;45:698-715.

149. Saini BS, Singh D, Uddin M, Kumar V. Improved power spectrum estimation for RR-interval time series. World Acad Sci Eng Technol. 2008:46:44-8. 
150. Singh D, Vinod K, Saxena SC, Deepak KK. Effects of RR segment duration on HRV spectrum estimation. Physiol Meas. 2004;25:721.

151. Burr RL, Cowan MJ. Autoregressive spectral models of heart rate variability. Practical issues. J Electrocardiol. 1992;25(Suppl):224-33.

152. Moody GB. Spectral analysis of heart rate without resampling. In: Proceedings of Computers in Cardiology Conference. Washington, D.C.: IEEE; 1993. p. 715-8.

153. Chang KL, Monahan KJ, Griffin MP, Lake D, Moorman JR. Comparison and clinical application of frequency domain methods in analysis of neonatal heart rate time series. Ann Biomed Eng. 2001;29:764-74.

154. Clifford GD, Tarassenko L. Quantifying errors in spectral estimates of HRV due to beat replacement and resampling. IEEE Trans Biomed Eng. 2005:52:630-8.

155. Mukherjee S, Yadav R, Yung I, Zajdel DP, Oken BS. Sensitivity to mental effort and test-retest reliability of heart rate variability measures in healthy seniors. Clin Neurophysiol. 2011;122:2059-66.

156. Delane A, Bohorquez J, Gupta S, Schiavenato M. Lomb algorithm versus fast Fourier transform in heart rate variability analyses of pain in premature infants. Conf Proc IEEE Eng Med Biol Soc. 2016;2016:944-7.

157. Thong T, Yung IO, P Zajdel DP, Ellingson RM, McNames J, Aboy M, et al. Heart rate variability analysis of effect of nicotine using periodograms. Conf Proc IEEE Eng Med Biol Soc. 2004;1:294-7.

158. Van Dongen HP, Olofsen E, VanHartevelt JH, Kruyt EW. Searching for biological rhythms: peak detection in the periodogram of unequally spaced data. J Biol Rhythm. 1999;14:617-20.

159. Tarvainen MP, Niskanen J-P, Lipponen JA, Ranta-aho PO, Karjalainen PA. Kubios HRV - Heart rate variability analysis software. Comput Methods Prog Biomed. 2014;113:210-20.

160. Pan J, Tompkins WJ. A real-time QRS detection algorithm. IEEE Trans Biomed Eng. 1985;32:230-6.

161. Tarvainen M, Lipponen J, Niskanen J-P, Ranta-aho P. Kubios HRV version 3.1 User's Guide. 2018. http://www.kubios.com/downloads/Kubios_HRV_ Users_Guide.pdf.

162. Rincon Soler Al, Silva LEV, Fazan R, Murta LO. The impact of artifact correction methods of RR series on heart rate variability parameters. J Appl Physiol. 2017;124:646-52.

163. Tarvainen MP, Ranta-aho PO, Karjalainen PA. An advanced detrending method with application to HRV analysis. IEEE Trans Biomed Eng. 2002;49:172-5.

164. Bilo G, Zorzi C, Ochoa Munera JE, Torlasco C, Giuli V, Parati G. Validation of the Somnotouch-NIBP noninvasive continuous blood pressure monitor according to the European Society of Hypertension International Protocol revision 2010. Blood Press Monit. 2015;20:291-4.

165. O'Brien E, Atkins N, Stergiou G, Karpettas N, Parati G, Asmar R, et al. European Society of Hypertension International Protocol revision 2010 for the validation of blood pressure measuring devices in adults. Blood Press Monit. 2010;15:23-38.

166. Verdecchia P, Angeli F, Borgioni C, Gattobigio R, Reboldi G. Ambulatory blood pressure and cardiovascular outcome in relation to perceived sleep deprivation. Hypertension. 2007:49:777-83.

167. Séi H. Blood pressure surges in REM sleep: A mini review. Pathophysiology. 2012;19:233-41.

168. Palagini L, Moretto U, Dell'Osso L, Carney C. Sleep-related cognitive processes, arousal, and emotion dysregulation in insomnia disorder: the role of insomnia-specific rumination. Sleep Med. 2017;30:97-104.

169. Karlson CW, Stevens NR, Olson CA, Hamilton NA. Depression, fatigue, and presleep arousal: a mediation model. J Coll Stud Psychother. 2010;24:307-27.

170. Gieselmann A, de Jong-Meyer R, Pietrowsky R. Kognitive und körperliche Erregung in der Phase vor dem Einschlafen. Z Für Klin Psychol Psychother. 2012:41:73-80.

171. Riemann D, Baum E, Cohrs S, Crönlein T, Hajak G, Hertenstein E, et al. S3Leitlinie Nicht erholsamer Schlaf/Schlafstörungen. Somnologie. 2017:21:2-44.

172. Görtelmeyer R, Schlaffragebogen A und B. SF-A/R und SF-B/R: Hogrefe; 2011.

173. Hoddes E, Zarcone V, Smythe H, Phillips R, Dement WC. Quantification of sleepiness: a new approach. Psychophysiology. 1973;10:431-6.

174. MacLean AW, Fekken GC, Saskin P, Knowles JB. Psychometric evaluation of the Stanford Sleepiness Scale. J Sleep Res. 1992;1:35-9.

175. Morgenthaler T, Kramer M, Alessi C, Friedman L, Boehlecke B, Brown T, et al. Practice parameters for the psychological and behavioral treatment of insomnia: an update. An american academy of sleep medicine report. Sleep. 2006:29:1415-9.
176. Horvath P. Treatment expectancy as a function of the amount of information presented in therapeutic rationales. J Clin Psychol. 1990;46:636-42.

177. Rutherford BR, Wager TD, Roose SP. Expectancy and the treatment of depression: a review of experimental methodology and effects on patient outcome. Curr Psychiatr Rev. 2010;6:1-10.

178. Devilly GJ, Borkovec TD. Psychometric properties of the credibility/ expectancy questionnaire. J Behav Ther Exp Psychiatry. 2000;31:73-86.

179. Williams AD, Blackwell SE, Mackenzie A, Holmes EA, Andrews G. Combining imagination and reason in the treatment of depression: a randomized controlled trial of internet-based cognitive-bias modification and internetCBT for depression. J Consult Clin Psychol. 2013:81:793.

180. O'Callaghan CA. OxMaR: open source free software for online minimization and randomization for clinical trials. PLoS One. 2014;9:e110761.

181. Haskell WL, Lee I-M, Pate RR, Powell KE, Blair SN, Franklin BA, et al. Physical activity and public health: updated recommendation for adults from the American College of Sports Medicine and the American Heart Association. Circulation. 2007;116:1081-93.

182. Abele-Brehm A, Brehm W. Zur Konzeptualisierung und Messung von Befindlichkeit: Die Entwicklung der "Befindlichkeitsskalen" (BFS) [The conceptualization and measurement of mood: The development of the "Mood Survey."]. Diagnostica. 1986;32:209-28.

183. Kellmann M, Golenia M. Skalen zur Erfassung der aktuellen Befindlichkeit im Sport. Dtsch Z Sportmed. 2003;54:329-30.

184. Höchsmann C, Knaier R, Eymann J, Hintermann J, Infanger D, SchmidtTrucksäss A. Validity of activity trackers, smartphones, and phone applications to measure steps in various walking conditions. Scand J Med Sci Sports. 2018;28(7):1818-1827.

185. Castor Electronic Data Capture. Amsterdam: Ciwit BV; 2018.

186. Kahan BC, Morris TP. Reporting and analysis of trials using stratified randomisation in leading medical journals: review and reanalysis. BMJ. 2012;345:e5840.

187. Borm GF, Fransen J, Lemmens WAJG. A simple sample size formula for analysis of covariance in randomized clinical trials. J Clin Epidemiol. 2007;60:1234-8

188. Youngstedt SD. Ceiling and floor effects in sleep research. Sleep Med Rev. 2003;7:351-65.

189. Rubio-Arias JÁ, Marín-Cascales E, Ramos-Campo DJ, Hernandez AV, PérezLópez FR. Effect of exercise on sleep quality and insomnia in middle-aged women: A systematic review and meta-analysis of randomized controlled trials. Maturitas. 2017;100:49-56.

190. Follmann D, Elliott $P$, Suh I, Cutler J. Variance imputation for overviews of clinical trials with continuous response. J Clin Epidemiol. 1992;45:769-73.

191. Rosenthal R. Meta-analytic procedures for social research. Beverly Hills: CA: Sage; 1991.

192. Stubbs B, Vancampfort D, Rosenbaum S, Ward PB, Richards J, Soundy A, et al. Dropout from exercise randomized controlled trials among people with depression: A meta-analysis and meta regression. J Affect Disord. 2016:190:457-66

193. Su Y-S, Gelman A, Hill J, Yajima M. Multiple imputation with diagnostics (mi) in R: Opening windows into the black box. J Stat Softw. 2011:45:1-31.

194. Benjamini Y, Hochberg Y. Controlling the false discovery rate: a practical and powerful approach to multiple testing. J R Stat Soc Ser B Methodol. 1995:57(1):289-300.

\section{Publisher's Note}

Springer Nature remains neutral with regard to jurisdictional claims in published maps and institutional affiliations.

Ready to submit your research? Choose BMC and benefit from:

- fast, convenient online submission

- thorough peer review by experienced researchers in your field

- rapid publication on acceptance

- support for research data, including large and complex data types

- gold Open Access which fosters wider collaboration and increased citations

- maximum visibility for your research: over $100 \mathrm{M}$ website views per year

At $\mathrm{BMC}$, research is always in progress.

Learn more biomedcentral.com/submissions 